\title{
Equation-of-state dependent features in shock-oscillation modulated neutrino and gravitational-wave signals from supernovae
}

\author{
A. Marek, H.-T. Janka, and E. Müller
}

\begin{abstract}
Max-Planck-Institut für Astrophysik, Karl-Schwarzschild-Str.1, 85748 Garching, Germany
e-mail: thj@mpa-garching.mpg.de
\end{abstract}

Received 29 August 2008 / Accepted 28 January 2009

\begin{abstract}
We present two-dimensional (axisymmetric) neutrino-hydrodynamic simulations of the long-time accretion phase of a $15 M_{\odot}$ progenitor star after core bounce and before the launch of a supernova explosion, when non-radial hydrodynamic instabilities like convection occur in different regions of the collapsing stellar core and the standing accretion shock instability (SASI) leads to large-amplitude oscillations of the stalled shock with a period of tens of milliseconds. Our simulations were performed with the PROMETHEUS-VERTEX code, which includes a multi-flavor, energy-dependent neutrino transport scheme and employs an effective relativistic gravitational potential. Testing the influence of a stiff and a soft equation of state for hot neutron star matter, we find that the non-radial mass motions in the supernova core impose a time variability on the neutrino and gravitational-wave signals with larger amplitudes, as well as higher frequencies in the case of a more compact nascent neutron star. After the prompt shock-breakout burst of electron neutrinos, a more compact accreting remnant produces higher neutrino luminosities and higher mean neutrino energies. The observable neutrino emission in the SASI sloshing direction exhibits a modulation of several ten percent in the luminosities and around $1 \mathrm{MeV}$ in the mean energies with most power at typical SASI frequencies between roughly 20 and $100 \mathrm{~Hz}$. The modulation is caused by quasi-periodic variations in the mass accretion rate of the neutron star in each hemisphere. At times later than $\sim 50-100 \mathrm{~ms}$ after bounce, the gravitational-wave amplitude is dominated by the growing low-frequency $(\$ 200 \mathrm{~Hz})$ signal associated with anisotropic neutrino emission. A high-frequency wave signal results from nonradial gas flows in the outer layers of the anisotropically accreting neutron star. Right after bounce such nonradial mass motions occur due to prompt post-shock convection in both considered cases and contribute mostly to the early wave production around $100 \mathrm{~Hz}$. Later they are instigated by the SASI and by convective overturn that vigorously stir the neutrino-heating and cooling layers, and also by convective activity developing below the neutrinosphere. The gravitational-wave power then peaks at about $300-800 \mathrm{~Hz}$, connected to changes in the mass quadrupole moment on a timescale of milliseconds. Distinctively higher spectral frequencies originate from the more compact and more rapidly contracting neutron star. Both the neutrino and gravitational-wave emission therefore carry information that is characteristic of the properties of the nuclear equation of state in the hot remnant. The detectability of the SASI effects in the neutrino and gravitational-wave signals is briefly discussed.
\end{abstract}

Key words. stars: supernovae: general - hydrodynamics - neutrinos - gravitational waves - dense matter

\section{Introduction}

Neutrinos and gravitational waves are the most direct potential probes of the processes that occur deep inside of a dying star, accompanying or causing the initiation of the stellar explosion. Neutrinos were already detected in connection with supernova SN 1987A (Bionta et al. 1987; Hirata et al. 1987; Alexeyev et al. 1988), although with poor statistics so that the extraction of information for constraining the explosion mechanism was not possible. The simultaneous measurement of signals of both types remains a very realistic hope for the next Galactic supernova.

Capturing neutrinos and gravitational waves from the same source has the advantage of providing complementary insight into the conditions of the stellar core. While neutrino signals reflect the density structure and thermodynamic conditions in the high-density plasma of the collapsing core and forming neutron star, gravitational waves carry crucial information about the dynamics and nonradial motions of the stellar matter, e.g. of its rotational state or of hydrodynamic instabilities such as convection that deform and stir the condensing central compact remnant of the explosion.

The prompt burst of electron neutrinos, for example, signals the breakout of the supernova shock from the neutrinosphere and the shock heating of matter in this region. The neutrino emission after core bounce is a sensitive probe of the mass accretion rate on the forming neutron star, hence of the density structure of the infalling layers of the dying star (e.g., Liebendörfer et al. 2003; Buras et al. 2006b). The onset of the explosion is expected to show up as a more or less sudden drop in the mass accretion rate of the forming neutron star. A soft supernuclear equation of state will lead to a more compact and hotter remnant, radiating higher neutrino luminosities and more energetic neutrinos (e.g., Janka et al. 2005; Marek 2007). And a possible phase transition to nonnucleonic matter in the neutron star core may impose characteristic features like a second neutrino burst (Sagert et al. 2008) or, if triggering the collapse to a black hole, may cause an abrupt termination of the neutrino emission (e.g., Burrows 1988; Keil \& Janka 1995; Sumiyoshi et al. 2006, 2007; Fischer et al. 2008). 
Theoretical work on the gravitational-wave signals from stellar core collapse and explosion has a long history of successively refined numerical models. In particular the infall and bounce phases, which are theoretically relatively well understood parts of the evolution, have received a lot of interest, because a strong and characteristic signature could make them a promising source of a detectable gravitational-wave burst (for a review-like introduction to the subject and a nearly complete list of publications, see Dimmelmeier et al. 2008). For this to be the case, the core of the progenitor star must develop a sufficiently large deformation during its infall, and for that it must rotate enough rapidly. This, however, does not seem to be compatible with predictions from the latest generation of stellar evolution models for the vast majority of massive stars, which are expected to have lost most of their angular momentum before collapse (Heger et al. 2005). Only in rare, very special cases, possibly accounting for the few tenths of a percent of all stellar core collapses that produce gamma-ray bursts, such stars seem to be able to retain a high angular momentum in their core and to thus produce relativistic jets and highly asymmetric and extremely energetic, probably magnetohydrodynamically driven explosions (for a recent review, see e.g. Woosley \& Bloom 2006). If this hypothetical connection was true, the core bounce phase would not really offer grand perspectives for the measurement of gravitational waves.

In contrast, only relatively little work has so far been done on determining the wave signals from the post-bounce evolution of a supernova, although these signals are likely to carry important information about the still incompletely understood explosion mechanism and the associated core dynamics (see the review by Ott 2008). Müller \& Janka (1997) and later Müller et al. (2004) on the basis of significantly improved numerical models, showed that in the case of delayed, neutrino-driven explosions convective overturn behind the stalled shock as well as convection inside the nascent neutron star can account for sizable gravitational-wave signals, which should be detectable with a high probability from a Galactic supernova when the Advanced Laser Interferometer Gravitational-Wave Observatory (LIGO II) is running (see also Fryer et al. 2002, 2004).

While the delayed neutrino-heating mechanism relies on the support by strong nonradial hydrodynamic instabilities in the region of neutrino-energy deposition, magnetohydrodynamic explosions, if linked to rapid rotation, are expected to develop relatively soon after core bounce and thus to occur faster than the growth of the mentioned nonradial instabilities. They tap the reservoir of differential rotation in a collapsing stellar environment and therefore require rapidly spinning cores (see Burrows et al. 2007b; Thompson et al. 2005). Thus they are good candidates for sizable gravitational-wave pulses from the moment of core bounce (e.g., Kotake et al. 2006; Ott et al. 2004). The newly proposed acoustic explosion mechanism, in which largeamplitude core gravity-mode oscillations of the neutron star convert accretion power to pressure and shock waves that feed the supernova shock with acoustic energy (Burrows et al. 2006, $2007 a)$, is predicted to be associated with enormous and very characteristic gravitational-wave activity due to the fast periodic movement of roughly a solar mass of dense matter at late times ( 1 s) after core bounce (Ott et al. 2006).

In the present paper our focus is on an analysis of the features in the neutrino and gravitational-wave signals that might provide evidence for the action of the so-called standing accretion shock instability (SASI), which has been shown to lead to low- $\ell$ mode (in terms of an expansion in spherical harmonics with order $\ell$ ), large-amplitude nonradial shock deformation and violent sloshing motions of the stalled supernova shock
(Blondin et al. 2003; Blondin \& Mezzacappa 2007; Bruenn et al. 2006; Scheck et al. 2004, 2006, 2008; Ohnishi et al. 2006; Foglizzo et al. 2007; Yamasaki \& Foglizzo 2008). This SASI activity is found to reach the nonlinear regime at roughly $100 \mathrm{~ms}$ after core bounce and to grow in amplitude over possibly hundreds of milliseconds (Scheck et al. 2008; Marek \& Janka 2007; Burrows et al. 2006, 2007a). It does not only act as seed of powerful secondary convection but can also provide crucial aid for the neutrino-heating mechanism by pushing the accretion shock to larger radii and by thus stretching the time accreted matter is exposed to neutrino heating in the gain layer (Buras et al. 2006b; Scheck et al. 2008; Marek \& Janka 2007; Murphy \& Burrows 2008). Moreover, the SASI is found to play the driving force of the g-modes pulsations of the neutron star core that are the essential ingredient of the acoustic mechanism.

Observational signatures of the presence of the SASI would therefore be extremely important for our understanding of how massive stars begin their explosion. Supernova asymmetries and pulsar kicks are one, yet not unambiguous observational hint. Neutrinos and gravitational waves, which originate directly from the region where the blast is initiated, however, may remain the only way to obtain direct information. It is therefore a highly relevant question to ask whether any characteristic structures are imprinted on the neutrino and gravitational-wave emission by the SASI activity in the supernova core. In contrast to convection, which exhibits the fastest growth for the higher- $\ell$ modes (see Foglizzo et al. 2006), the SASI is expected to possess the largest growth rates for the dipolar and quadrupolar deformations (corresponding to $\ell=1,2$; Blondin \& Mezzacappa 2006; Foglizzo et al. 2007; Yamasaki \& Foglizzo 2008; Foglizzo 2001, 2002). Even in the fully nonlinear situation the geometry and motion of the shock and post-shock layer are found to be governed by these lowest modes.

We present here an analysis of the neutrino and gravitationalwave signals that are calculated on the basis of the twodimensional post-bounce and pre-explosion simulations of a $15 M_{\odot}$ star recently published by Marek \& Janka (2007). We constrain ourselves to two nonrotating models, which allow us to discuss the differences that can be expected from a stiff and a soft nuclear equation of state (results of the corresponding 1D simulations can also be found in Marek \& Janka 2007). We find that the SASI sloshing of the shock and the associated quasi-periodic mass motions lead to a time-modulation of the neutrino emission and to gravitational-wave amplitudes whose size and characteristic frequency depend on the compactness of the proto-neutron star during the first half of a second after core bounce. Though the SASI contributions to the power spectra are superimposed by a significant high-frequency "noise" due to convective fluctuations, at least the combination of measurements should make it possible to identify the SASI activity in the supernova core.

Our paper is organized as follows. In Sect. 2 we will briefly outline the main numerical and physics ingredients of our simulations, and the basic features of the two simulations we compare. In Sect. 3 we discuss our results with respect to the SASI effects on the shock motion, neutrino emission, and gravitational-wave signal, and in Sect. 4 we will summarize our findings and draw conclusions, including a discussion whether the SASI modulations are detectable by neutrino and gravitational-wave experiments.

\section{Code, input, models}

The 2D simulations discussed in this paper were performed with the PROMETHEUS-VERTEX code, whose numerical aspects 
and the implemented microphysics were described by Rampp \& Janka (2002) and Buras et al. (2006a), and the publications quoted in those papers. The detailed list of ingredients was also provided by Marek \& Janka (2007), where the simulations were already introduced as Models M15LS-2D and M15HW-2D and compared with other cases in a greater set of calculations. We therefore repeat only a few essential aspects of immediate relevance here and refer the reader to Sect. 2 of the Marek \& Janka (2007) paper for more complete information.

The hydrodynamics module of the code is based on a conservative and explicit Eulerian implementation of a Godunovtype scheme with higher order spatial and temporal accuracy. It solves the nonrelativistic equations of motions for the stellar fluid, whose self-gravity is described by an "effective relativistic potential" for an approximative treatment of general relativistic gravity (see Marek et al. 2006, the discussed simulations were performed with the potential of Case A from this work). The neutrino transport, which is coupled to the hydrodynamics part via lepton number, energy, and momentum source terms, is computed with our "ray-by-ray plus scheme" (see Buras et al. 2006a). It accounts for the full neutrino-energy dependence of the transport but treats its dependence on the direction of the neutrino momentum in an approximative way, which is numerically less demanding and more efficient than a full multi-dimensional version of the transport. Recent multi-angle simulations in twodimensional situations (Ott et al. 2008) - though done at the expense of a sophisticated description of the energy dependence - show that a detailed angular treatment produces considerably less lateral smearing of the outward directed radiation field than flux-limited diffusion. We suspect that our ray-by-ray description compares much better with multi-angle results than fluxlimited diffusion does.

The progenitor star used for our simulations was model s15s7b2 from Woosley \& Weaver (1995), which is a standard nonrotating $15 M_{\odot}$ star widely used for supernova simulations. We employed two different nuclear equations of state $(E o S)$ for our studies: (1) a soft version of the Lattimer \& Swesty (1991) EoS ("L\&S EoS") with an incompressibility modulus of bulk nuclear matter of $180 \mathrm{MeV}$ and a symmetry energy parameter of $29.3 \mathrm{MeV}$, and (2) the considerably stiffer EoS of Hillebrandt \& Wolff (1985, "H\&W EoS"; see also Hillebrandt et al. 1984), whose parameter values are $263 \mathrm{MeV}$ and $32.9 \mathrm{MeV}$, respectively $^{1,2}$. The former leads to a radius of about $12 \mathrm{~km}$ for cold neutron stars with a "typical" (gravitational) mass of $1.4 M_{\odot}$, whereas this radius is roughly $14 \mathrm{~km}$ in the second case. The Hillebrandt \& Wolff EoS is based on a largely different modeling approach for inhomogeneous nucleon matter than the L\&S EoS and the more recent EoS of Shen et al. (1998). It employs a Hartree-Fock calculation in contrast to the compressible

\footnotetext{
1 Note that below a certain density, which is typically chosen to be $10^{11} \mathrm{~g} \mathrm{~cm}^{-3}$ after core bounce, we replace the high-density EoSs by an ideal-gas equation of state with electrons, positrons, photons, and a mixture of classical, nonrelativistic Boltzmann gases for nucleons, alpha particles, and 14 kinds of heavier nuclei (see Marek \& Janka 2007, for more information).

${ }^{2}$ In order to save computer time, in particular during the simulation phase right after core bounce when the timesteps are constrained to very low values, the model with the $H \& W$ EoS was performed with the assumption of equatorial symmetry until $125.3 \mathrm{~ms}$ post bounce. Only afterwards it was continued with a full $180^{\circ}$ grid. Tests showed that this had no important influence on the results, neither for the evolution of the shock radius, nor for the growth and development of hydrodynamic instabilities. In particular, we did not find any qualitative difference in the SASI modes, also for odd values of $\ell$, at later times (see Marek 2007).
}

liquid drop model of the L\&S EoS and the relativistic mean field description of the Shen et al. EoS. The three EoSs yield significantly different results in $1 \mathrm{D}$ core-collapse simulations with respect to the shock formation point, the luminosities and mean energies of the radiated neutrinos, and the evolution of the shock radius and neutron star radius after bounce (see Janka et al. 2005; Marek 2007; Figs. 6 and 7 in Janka et al. 2007). Since the EoS of Shen et al. yields intermediate values for many of these quantities, we consider the soft Lattimer \& Swesty EoS on the one hand and the stiff Hillebrandt \& Wolff EoS on the other as two cases that roughly span the range of extreme possibilities for baryonic matter around and above nuclear saturation density near core bounce and shortly afterwards.

\section{Results}

In the following we will compare our two simulations for these equations of state in detail, focussing on the observable signals whose properties depend on the asymmetries due to hydrodynamic instabilities in the supernova core after bounce.

\subsection{Hydrodynamic instabilities and shock motion}

Basically one can discriminate three regions and episodes of nonradial hydrodynamic instability in the stellar core after bounce: (i) prompt post-shock convection (Epstein 1979; Burrows \& Fryxell 1993; Janka \& Müller 1996; Müller \& Janka 1997; Swesty \& Myra 2005); (ii) convection inside the nascent neutron star, i.e. below the neutrinosphere of the electron neutrinos (Burrows 1987; Keil et al. 1996; Buras et al. 2006b; Dessart et al. 2006); and (iii) convective overturn in the neutrino-heating layer between the gain radius and the stalled supernova shock (Herant et al. 1994; Burrows et al. 1995; Janka \& Müller 1996; Fryer \& Warren 2002, 2004) as well as SASI activity (Blondin et al. 2003; Blondin \& Mezzacappa 2006; Ohnishi et al. 2006; Scheck et al. 2008). These regions can be identified for both 2D simulations in Fig. 1.

Region (i) arises when the newly formed supernova shock propagates outward through the infalling stellar iron core and experiences massive energy losses by nuclear photodisintegrations. This weakens the shock so that it leaves behind a layer with negative entropy gradient (before it encounters infalling material with even lower densities and therefore produces rising gas entropies again in spite of its further deceleration). In addition, a $Y_{\mathrm{e}}$ minimum is established near an enclosed mass of roughly $1 M_{\odot}$ when a large number of newly produced electron neutrinos begins to stream away from the neutrinosphere in a luminous shock-breakout burst. The electron fraction forms a trough around the radial position where the shock makes the transition from the neutrino-opaque to the neutrinotransparent regime (Fig. 2). The electron fraction decreases towards this minimum, because electron-capture neutrinos escape more slowly from regions with higher densities, and because the conversion of electrons to neutrinos proceeds more slowly at lower densities. In both simulations the negative entropy gradient overlaps partly with the negative $Y_{\mathrm{e}}$ gradient.

The layer where both the entropy and the $Y_{\mathrm{e}}$ gradients are negative is found to be Ledoux unstable (marked by the grey vertical bars in Fig. 2). This region lies between the first local maximum and the following local minimum of the entropy profile. It contains considerably more mass in the model computed with the $\mathrm{H} \& \mathrm{~W}$ EoS, where it is bounded by the mass coordinates of $0.63 M_{\odot}$ and $0.79 M_{\odot}$. At higher enclosed masses, the entropy rises slowly out of its local minimum. In contrast, in the 

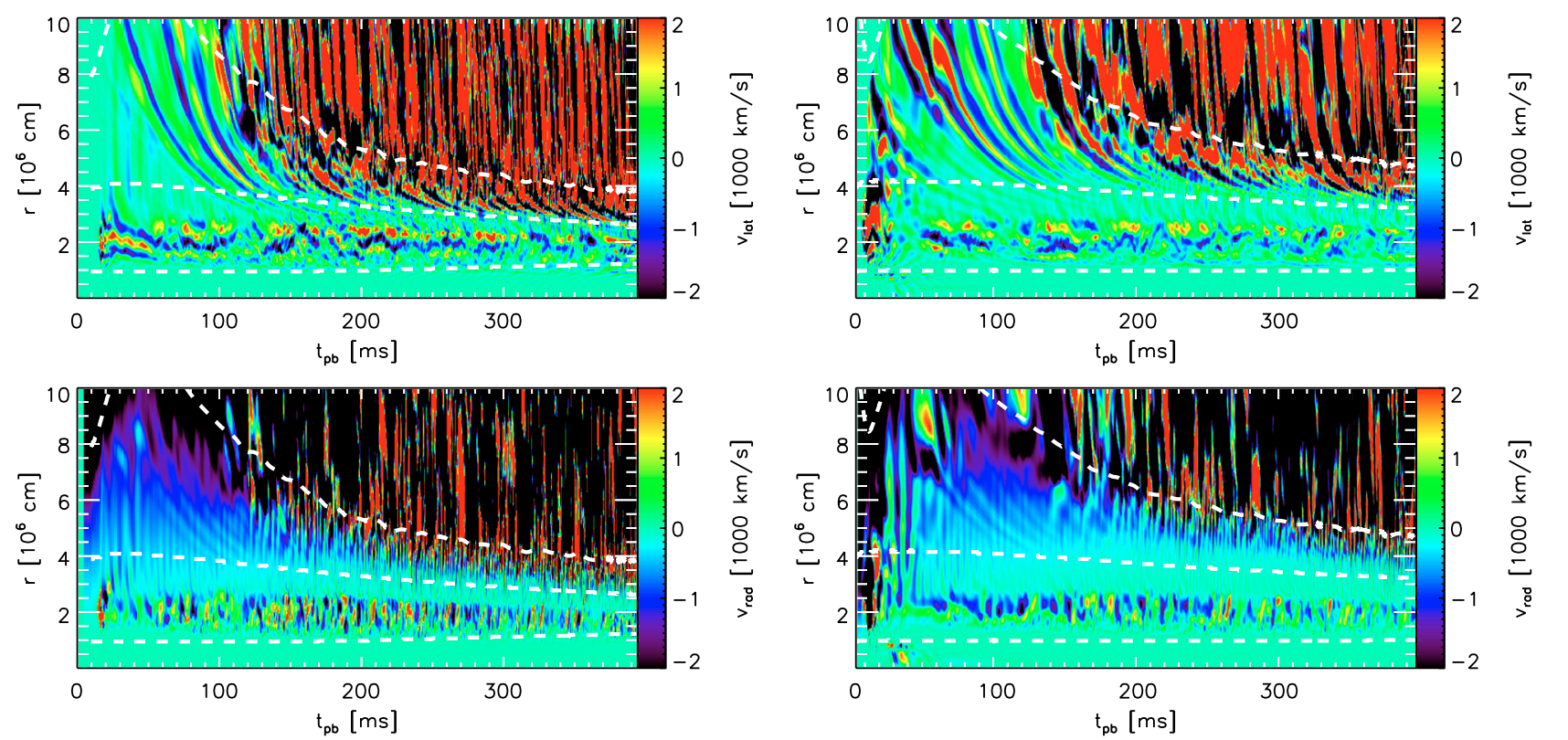

Fig. 1. Regions of mass motions due to hydrodynamic instabilities in the supernova core. The plots show color coded the lateral velocity (top) and the radial velocity (bottom) in the equatorial plane of the polar grid as functions of post-bounce time and radius for the 2D simulation with the L\&S EoS (left) and for the simulation with the H\&W EoS (right). The displayed range of velocity values is limited to $\pm 2 \times 10^{8} \mathrm{~cm} \mathrm{~s}^{-1}$. The white dashed lines mark (with decreasing radius) the locations of densities $10^{10}, 10^{12}$, and $10^{14} \mathrm{~g} \mathrm{~cm}^{-3}$. Prompt post-shock convection is strongest at $r \gtrsim 15 \mathrm{~km}$ before $t \sim 30 \mathrm{~ms}$ after core bounce, proto-neutron star convection occurs later at $r \lesssim 30 \mathrm{~km}$ and $\rho>10^{12} \mathrm{~g} \mathrm{~cm}^{-3}$, and SASI and convective activity behind the shock are visible at $r \gtrsim 30-40 \mathrm{~km}$.
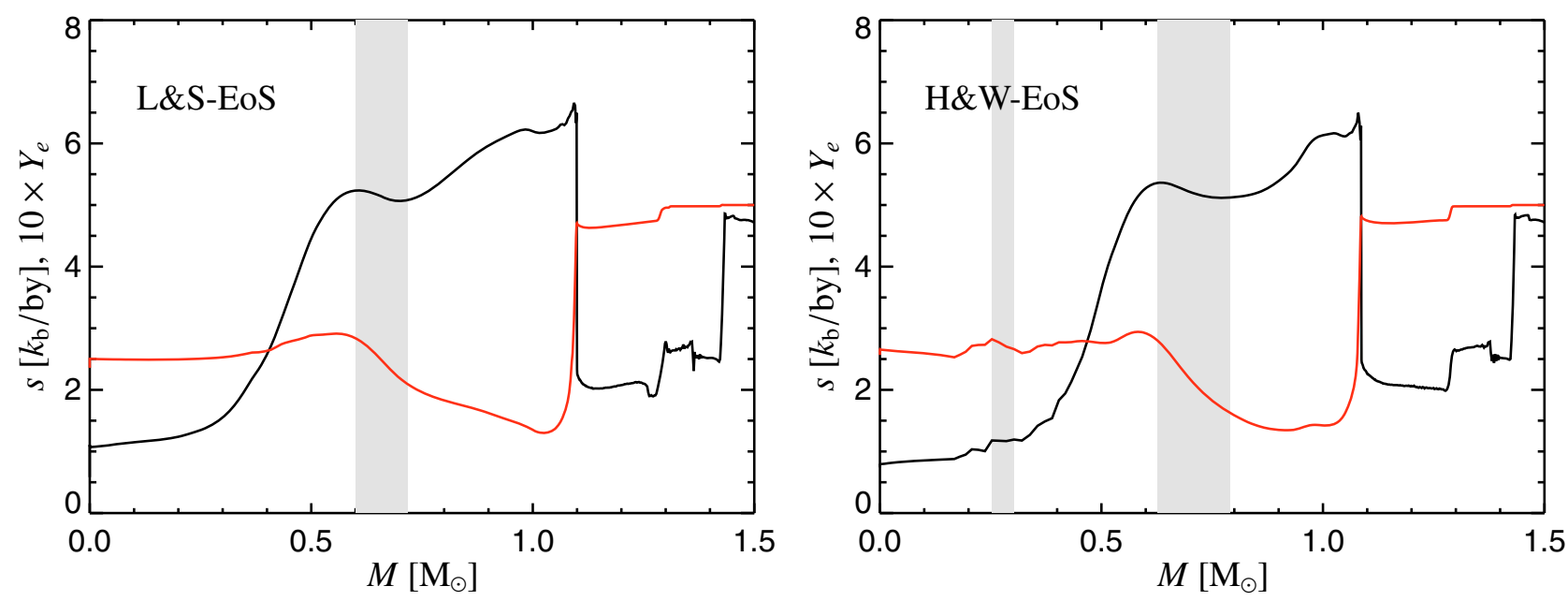

Fig. 2. Entropy (black) and electron fraction (red) profiles (the plotted values correspond to $10 \times Y_{\mathrm{e}}$ ) as functions of enclosed mass at $10 \mathrm{~ms}$ after core bounce in spherically symmetric counterparts of the two 2D simulations discussed in this paper. The shock is the entropy jump near $1.1 M_{\odot}$. The vertical grey bars indicate the mass regions that are convectively unstable according to the Ledoux criterion.

L\&S case the boundaries are at $0.60 M_{\odot}$ and $0.71 M_{\odot}$, and the increase of the entropy in the overlying shells is much steeper. In the H\&W model the thicker layer in mass corresponds also to a much wider radial domain at a larger distance from the stellar center. Because of higher growth rates the convective activity sets in more immediately after the shock passage and lateron becomes stronger and encompasses a greater fraction of the stellar core. This can be seen at $t \lesssim 30 \mathrm{~ms}$ after bounce in Fig. 1, where the righthand panels for the $\mathrm{H} \& \mathrm{~W}$ simulation in comparison to the lefthand panels show significantly more violent convective overturn. While the L\&S model develops visible mass motions between $\sim 15 \mathrm{~km}$ and $\sim 40 \mathrm{~km}$, the $\mathrm{H} \& \mathrm{~W}$ model shows stronger activity at radii of $15 \mathrm{~km} \lesssim r \lessgtr 80 \mathrm{~km}$.
After some $10 \mathrm{~ms}$ the profiles of entropy and electron fraction are flattened and with the disappearance of the driving force the convective activity begins to calm down (this can be recognized better in the two lower panels of Fig. 1). Now, however, region (ii) is being formed. Neutrino transport begins to reduce the electron fraction deeper inside the nascent neutron star. As a consequence of this the lower boundary of the $Y_{\mathrm{e}}$ trough and thus the negative $Y_{\mathrm{e}}$ gradient moves gradually inward. At the same time the energy losses by neutrinos reduce the level of the entropy plateau at $s \sim 5 k_{\mathrm{B}} /$ nucleon such that the plateau becomes wider and its inner edge also moves inward. These changes in the entropy and lepton number profiles in combination lead to the establishment (or/and maintenance) of a convectively 

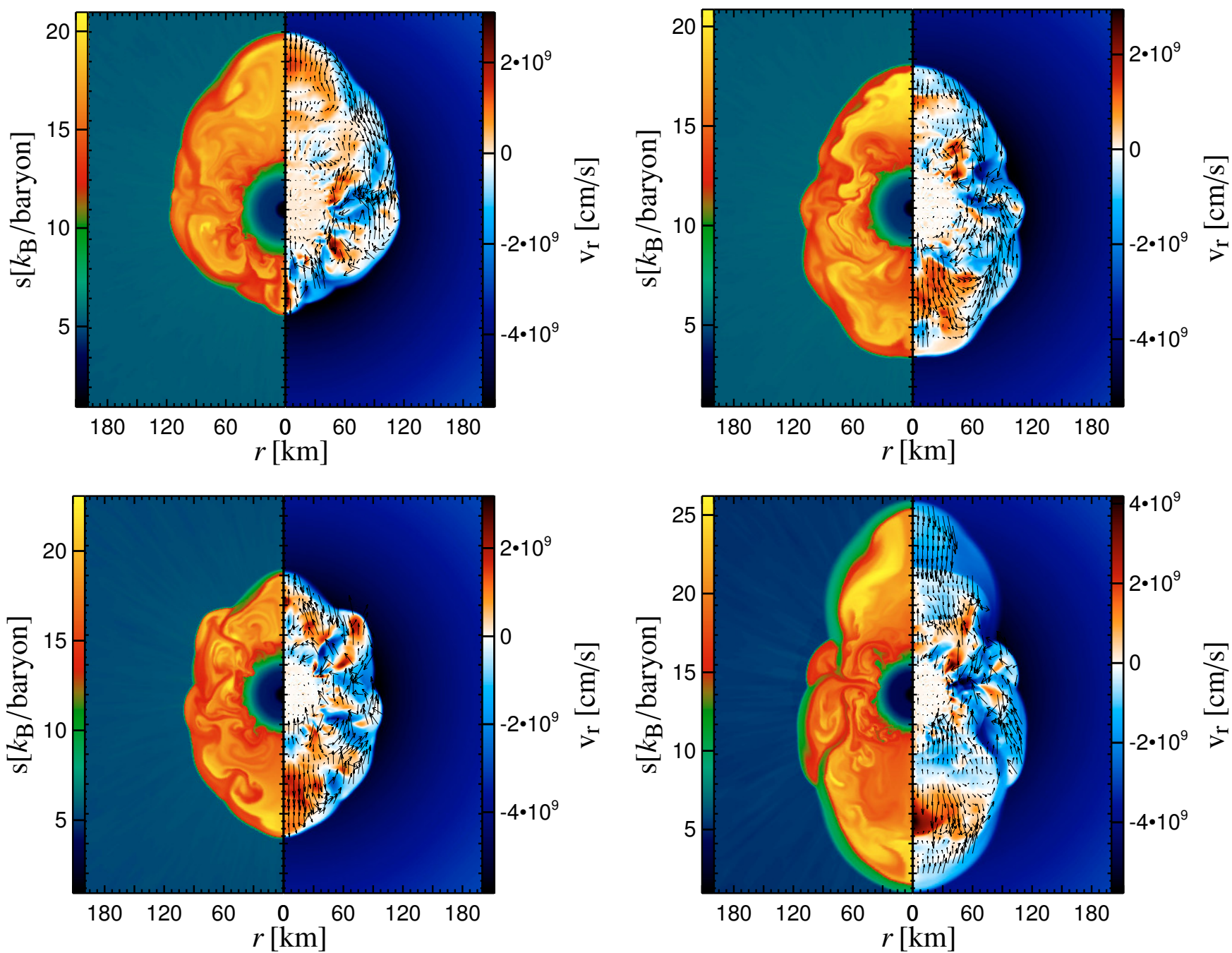

Fig. 3. Four representative snapshots from the 2D simulation with the L\&S EoS at post-bounce times of $247 \mathrm{~ms}$ (top left), $255 \mathrm{~ms}$ (top right), $322 \mathrm{~ms}$ (bottom left), and $375 \mathrm{~ms}$ (bottom right). The lefthand panel of each figure shows color-coded the entropy distribution, the righthand panel the radial velocity component with white and whitish hues denoting matter at or near rest; black arrows in the righthand panel indicate the direction of the velocity field in the post-shock region (arrows were plotted only in regions where the absolute values of the velocities were less than $2 \times 10^{9} \mathrm{~cm} \mathrm{~s}^{-1}$ ). The vertical axis is the symmetry axis of the 2D simulation. The plots visualize the accretion funnels and expansion flows in the SASI layer, but the chosen color maps are unable to resolve the convective shell inside the nascent neutron star.

unstable layer inside the proto-neutron star. About 50-60 ms after bounce, following a short, intermediate period of relative quiescence, both of our 2D models indeed show the reappearance of vivid overturn activity at densities above $10^{12} \mathrm{~g} \mathrm{~cm}^{-3}$ (Fig. 1). The convective transport of lepton number and entropy supports and enhances the mentioned structural changes so that a long-lasting zone with convective mixing develops (compare our Fig. 1 with Figs. 9 and 10 of Dessart et al. 2006). This zone encompasses a growing mass region within the broadening $Y_{\mathrm{e}}$ trough.

Region (iii) with nonradial hydrodynamic mass motions develops outside of the steep density gradient at the surface of the nascent neutron star, where the neutrinospheres of all neutrinos are located. First signs of low- $\ell$ mode, low-amplitude SASI sloshing can be seen here already some $10 \mathrm{~ms}$ after bounce. At about $100 \mathrm{~ms}$ after bounce this activity strengthens significantly because at this time neutrino energy deposition in the gain layer has begun to build up a negative entropy gradient between the gain radius and the stagnant supernova shock. The neutrino-heating region is unstable to convection, and the presence of convective motions produces vorticity and entropy perturbations that feed back into the advective-acoustic cycle that is considered as an explanation of the SASI phenomenon (see Foglizzo 2001, 2002; Foglizzo et al. 2007). Thus the onset of convection may lead to an amplification of the SASI growth. On the other hand, the violent shock oscillations that are characteristic of the fully developed SASI produce large entropy variations in the downstream region. These act as seeds for secondary convection (Scheck et al. 2008) and have been found to aid the neutrino-heating mechanism for powering supernova explosions (Scheck et al. 2008; Marek \& Janka 2007; see also Murphy \& Burrows 2008). In the nonlinear regime convective and SASI activity are inseparably coupled, and a high $\ell$ convective mode pattern occurs superimposed on the low $-\ell$ mode SASI deformations of the shock contour and of the post-shock region (for snapshots, see Fig. 3 and Marek \& Janka 2007; Scheck et al. 2008).

Although both simulations show the same basic features and evolutionary stages, the differences in details are interesting. 

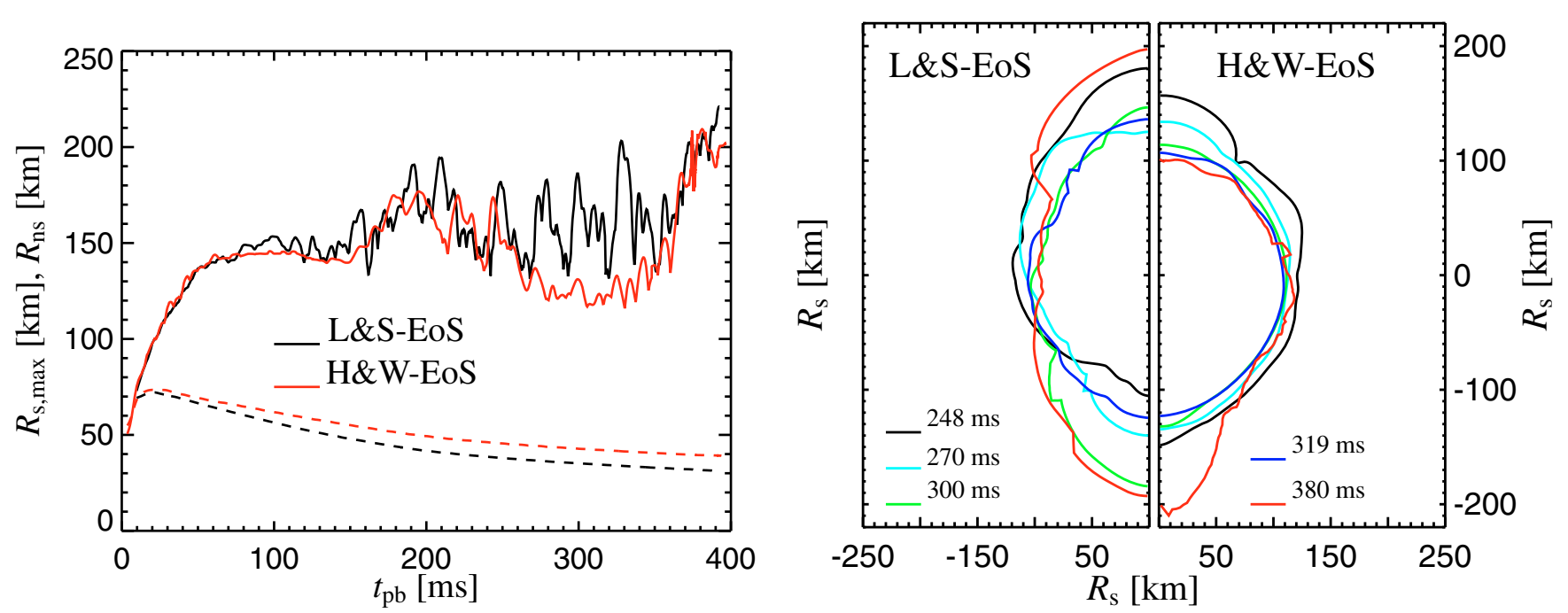

Fig. 4. Left: maximum shock radii (solid lines) and proto-neutron star radii (dashed lines) as functions of post-bounce time for the 2D simulations with different nuclear equations of state. The neutron star radii are determined as the locations where the rest-mass density is equal to $10^{11} \mathrm{~g} \mathrm{~cm}^{-3}$. Right: shock contours at the different post-bounce times listed in the figure. The vertical axis of the plot is the symmetry axis of the simulation.

As mentioned above, the H\&W model develops more vigorous prompt post-shock convection in a region with higher mass and wider radial extension. In contrast, the SASI activity in this model is appreciably less strong than that in the calculation with the L\&S EoS until roughly $350 \mathrm{~ms}$ after bounce. This can be seen in Fig. 4, where the maximum shock radius of the L\&S case exhibits significantly bigger SASI amplitudes and correspondingly the shock contours show more extreme nonspherical deformation (righthand panel of Fig. 4). These differences grow during the nonlinear phase of the SASI between $\sim 150$ and $350 \mathrm{~ms}$ until at $t \gtrsim 350 \mathrm{~ms}$ p.b. the SASI sloshing in the H\&W model also gains more power and the maximum shock radii of both simulations become more similar again.

We suspect that the more compact proto-neutron star for the softer L\&S EoS leads to conditions that favor strong SASI activity, possibly because of the more efficient neutrino heating (and more vigorous post-shock convection) as a consequence of the higher neutrino luminosities and harder neutrino spectra that are radiated from a more compact and hotter nascent neutron star (see Figs. 6 and 7 and Sect. 3.2). Another possible explanation in the context of the advective-acoustic cycle scenario may be different amplification factors of perturbations in the two models. Due to the lack of good theoretical insight into the behavior of the SASI in the fully nonlinear regime, we do not see a way how to facilitate deeper understanding by further analysis. For both suggested explanations, however, one might expect that in the H\&W simulation at late post-bounce times, when the protoneutron star radius has contracted (Fig. 4) and the neutrinoheating timescale has decreased (see Fig. 6 in Marek \& Janka 2007), the conditions for violent SASI activity have the tendency to improve. This would be consistent with our observed growth of the SASI amplitudes near the end of this simulation.

In Fig. 5, lefthand panels, we plot the time-evolution of the amplitudes $A_{1,0}, A_{2,0}$, and $A_{3,0}$, of the decomposition of the angle-dependent shock radius $R_{\mathrm{s}}(\theta, t)$ into spherical harmonics:

$R_{\mathrm{s}}(\theta, t) \equiv \sum_{\ell=0}^{\infty} A_{\ell, 0}(t) P_{\ell}^{0}(\cos \theta)$, where $m=0$ because of the axial symmetry of our models and $P_{\ell}^{0}(\cos \theta)$ are the Legendre polynomials ${ }^{3}$. Figure 5 confirms our description above: the amplitudes for the L\&S run are typically 2-3 times larger than those of the $\mathrm{H} \& \mathrm{~W}$ simulation. Only near the end of the computed evolution (at $t \gtrsim 350 \mathrm{~ms}$ p.b.), $A_{1,0}$ and $A_{3,0}$ of both models reach very similar values. This is not so for $A_{2,0}$, which exhibits oscillatory behavior on top of a clear slope to positive values in both simulations, signaling a trend in growing prolate deformation of the shock. Until the end of the computational runs, however, this deformation remains considerably more pronounced in the case of the model with the L\&S EoS.

The righthand panels of Fig. 5 provide the Fourier transforms of the time-dependent mode amplitudes. One can see a broad peak of the Fourier spectra between about 10 and roughly $100 \mathrm{~Hz}$, followed by a steep decline towards higher frequencies. The $\ell=1$ amplitude exhibits the clearest, jagged peak structure at a frequency of $20-60 \mathrm{~Hz}$ for the L\&S run, and a smoother peak at $30-40 \mathrm{~Hz}$ with a secondary one around $65 \mathrm{~Hz}$ for the $\mathrm{H} \& \mathrm{~W}$ model. $A_{2,0}$ shows most power at frequencies around $30-50 \mathrm{~Hz}$ and $70-90 \mathrm{~Hz}$ in both simulations, and $A_{3,0}$ possesses a broad Fourier maximum below $\sim 100-130 \mathrm{~Hz}$, with an indication of a peak at about $50 \mathrm{~Hz}$ in the $\mathrm{H} \& \mathrm{~W}$ case. There is no really clear correlation of the SASI oscillation period with the compactness of the nascent neutron star or with the average radius of the stalled shock except for, maybe, the slightly higher frequency of the main $\ell=1$ peak in the L\&S run. Because of the time-dependent structure of the accretor and of the whole postshock region the appearance of well localized and very prominent peaks in the spectrum might not be expected. In addition, shock wobbling associated with convective mass motions produces a background of short-wavelength "noise" at frequences up to roughly $200 \mathrm{~Hz}$ (Fig. 3). Accordingly, in particular the

\footnotetext{
3 Note that we have renormalized our amplitudes $A_{\ell, 0}$ compared to the coefficients $a_{\ell}$ used by Kotake et al. (2007) in order to directly display in Fig. 5 the radius variations associated with the different spherical harmonics components. The coefficient $A_{0,0}$ is identical with the angular average of the shock radius: $A_{0,0}(t)=\left\langle R_{\mathrm{s}}(t)\right\rangle \equiv(4 \pi)^{-1} \int \mathrm{d} \Omega R_{\mathrm{s}}(\theta, t)$, whose values and evolution are similar to those of the maximum shock radius plotted in Fig. 4.
} 

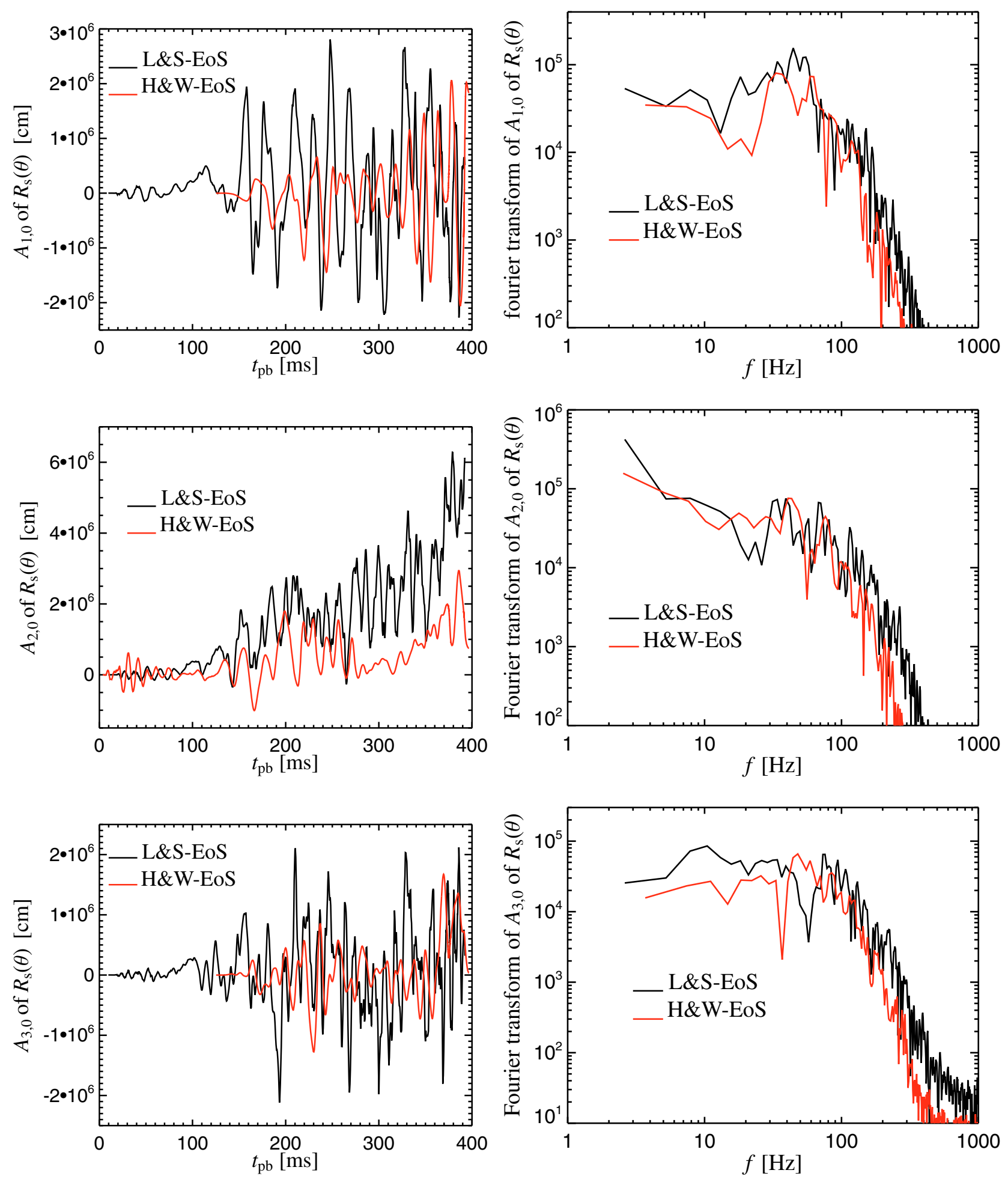

Fig. 5. Left: amplitudes for the decomposition of the angle-dependent position of the shock front, $R_{\mathrm{S}}(\theta, t)$, into spherical harmonic functions for the 2D simulation with the L\&S EoS (black lines) and with the H\&W EoS (red lines). Shown are the contributions of the modes for $(\ell, m)=(1,0)$ (top), $(2,0)$ (middle), and $(3,0)$ (bottom). The underlying trend in growing amplitudes for the $l=2$ mode towards the end of our simulations signals an increasing and persistent prolate deformation of the shock. Note that during the first $125.3 \mathrm{~ms}$ after core bounce, when the shock deformation is still relatively small, the simulation with the $\mathrm{H} \& \mathrm{~W}$ EoS was performed only with a $90^{\circ}$ wedge from the pole to the equator, and we can therefore plot only the amplitudes with even $\ell$ for this equatorially symmetric phase. Right: the Fourier transforms of the time-dependent mode amplitudes.

amplitudes for higher $\ell$ values show power nearly evenly distributed over a wide range of frequencies. The Fourier transforms for $A_{1,0}, A_{2,0}$, and $A_{3,0}$ plotted in Fig. 5 nevertheless confirm the stronger SASI activity in the simulation with the L\&S EoS: only for a few, narrow frequency windows the Fourier amplitudes of the $\mathrm{H} \& \mathrm{~W}$ model become larger than those of the L\&S run.

In the following two sections we will turn to an analysis of the consequences of the described nonradial hydrodynamic instabilities, in particular also of the SASI, for observable 

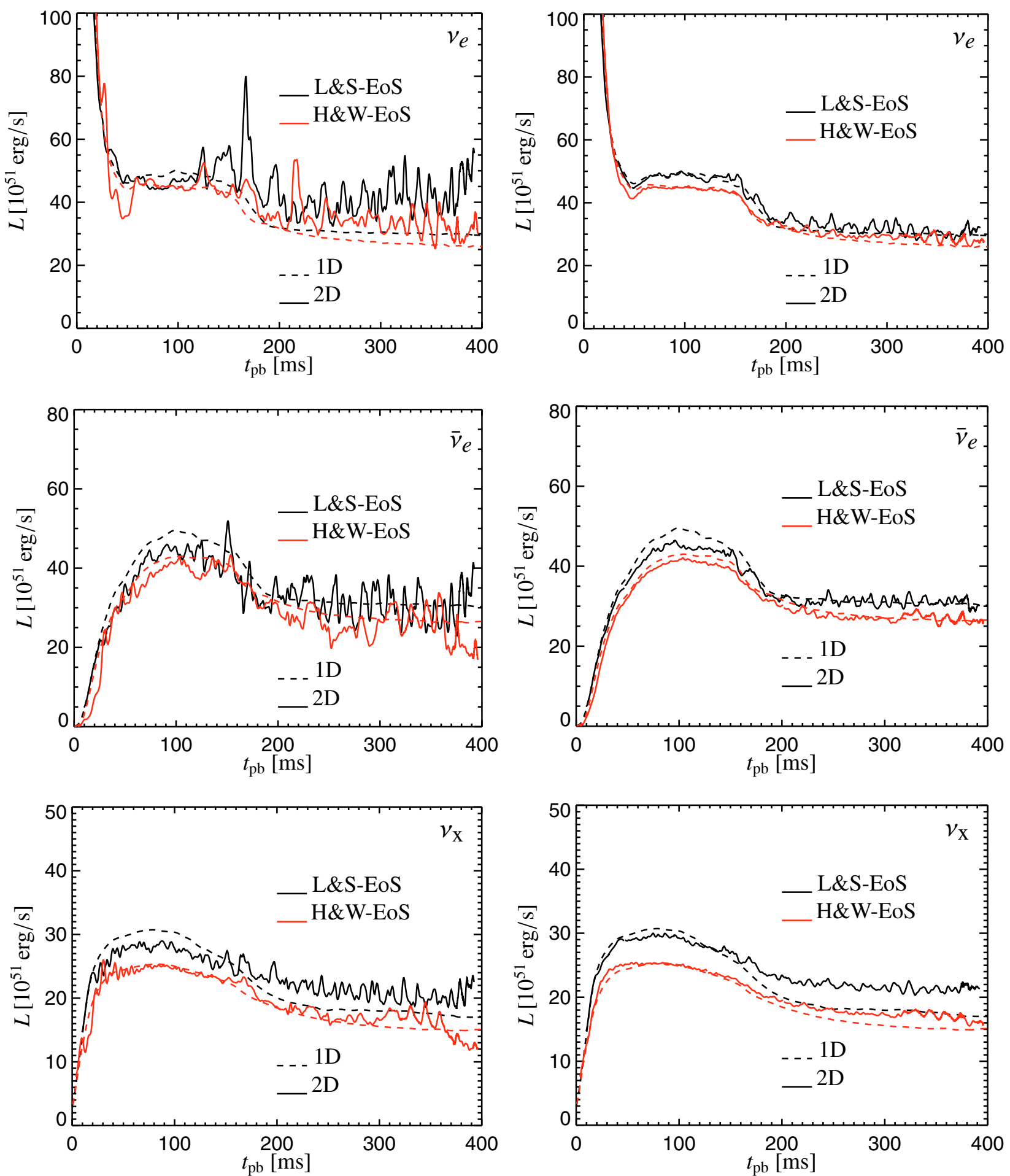

Fig. 6. Isotropic equivalent luminosities of electron neutrinos (top), electron antineutrinos (middle), and one kind of heavy-lepton neutrinos ( $v_{\mu}$, $\bar{v}_{\mu}, v_{\tau}$, or $\bar{v}_{\tau}$; bottom) versus time after core bounce as measurable for a distant observer located along the polar axis of the 2D spherical coordinate grid (solid lines). The dashed lines display the radiated luminosities of the corresponding spherically symmetric (1D) simulations. The evaluation was performed at a radius of $400 \mathrm{~km}$ (from there the remaining gravitational redshifting to infinity is negligible) and the results are given for an observer at rest relative to the stellar center. While the left column shows the (isotropic equivalent) luminosities computed from the flux that is radiated away in an angular grid bin very close to the north pole, the right column displays the emitted (isotropic equivalent) luminosities when the neutrino fluxes are integrated over the whole northern hemisphere of the grid (see Eqs. (2) and (4), respectively).

signals from the supernova core. One question of interest will be, whether the differences caused by the use of stiff or soft neutron star equations of state manifest themselves in some distinctive features of the signals.

\subsection{Neutrino emission}

The luminosities for neutrinos and antineutrinos of all flavors and for both 2D simulations, compared to the results of the corresponding 1D models, are given as functions of post-bounce time 

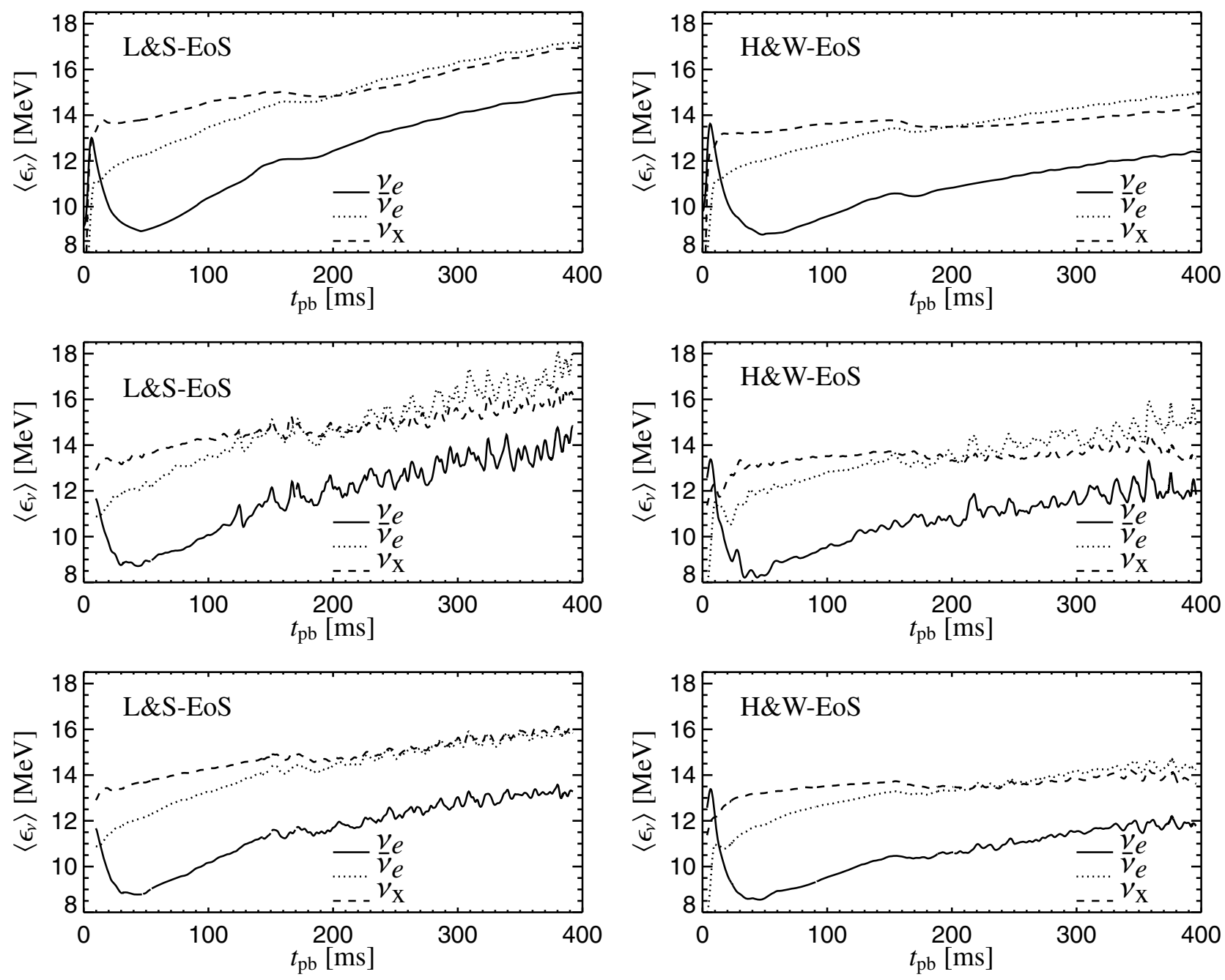

Fig. 7. Mean energies of radiated neutrinos as functions of post-bounce time for our 1D simulations (top) and 2D models (middle and bottom) with both equations of state (the lefthand panels are for the L\&S EoS, the right ones for the H\&W EoS). The displayed data are defined as ratios of the energy flux to the number flux and correspond to the luminosities plotted with dashed and solid lines in Fig. 6. The panels in the middle show results for a lateral grid zone near the north polar axis, the bottom panels provide results that are averaged over the whole northern hemisphere of the computational grid. In all cases the evaluation has been performed in the laboratory frame at a distance of $400 \mathrm{~km}$ from the stellar center.

in Fig. 6 (muon and tau neutrinos and their antiparticles, which we sometimes denote with $v_{x}$, are treated in the same way, and the plots show the luminosities of one individual type of these heavy-lepton neutrinos). The luminosities displayed there are actually not defined as the total rate of energy loss of the supernova core through neutrinos radiated in all directions. Instead, they represent the isotropic equivalent luminosities inferred by an observer at rest relative to the stellar center, viewing the source from the direction of the polar axis of the grid and from a large distance above the north pole (i.e., gravitational redshifting is taken into account). The results for an observer above the south pole look qualitatively and quantitatively very similar and lead to the same conclusions.

One of the goals of the present work is an analysis of luminosity fluctuations that are associated with modulations of the mass accretion rate of the nascent star due to SASI oscillations and convective overturn in the post-shock layer, which become very vigorous and create nonstationary conditions at later postbounce times (see Sect. 3.1 and Figs. 4 and 5). Strong shock retraction leads to a transient increase of the gas flow towards the neutron star and to the compression and enhanced cooling of the matter near the neutron star surface. In contrast, shock expansion has the opposite effect because it causes a deceleration of the infall or even outward acceleration of material that is accreted through the shock. Thus shock expansion stretches the time this matter remains in the gain layer and does not cool by neutrino emission (Marek \& Janka 2007; Scheck et al. 2008; Murphy \& Burrows 2008). Corresponding quasi-periodic fluctuations of the neutrino luminosities, strongest for electron neutrinos $v_{\mathrm{e}}$ and antineutrinos $\bar{v}_{\mathrm{e}}$ (which are more abundantly produced in the layer of freshly accreted, hot matter near the neutron star surface) can be seen in all panels of Fig. 6 .

In the lefthand panels of Fig. 6 we have constructed the isotropic equivalent luminosities from the neutrino radiation leaving the stellar core in one angular grid bin near the north pole of the mesh, i.e.,

$L_{v, 1}\left(\theta_{j+\frac{1}{2}}, t\right)=4 \pi r^{2} F_{v}\left(r, \theta_{j+\frac{1}{2}}, t\right)$

for $F_{v}\left(r, \theta_{j+\frac{1}{2}}, t\right)$ being the energy-integrated neutrino energy flux in the rest frame at the cell-centered (pole-near) latitudinal angle $\theta_{j+\frac{1}{2}}$ of the polar grid of the simulation, at time $t$ and a radius $r$ that is large enough such that the luminosity is conserved at greater radial distances (for the numerical evaluation we have chosen $r=400 \mathrm{~km}$ ). In contrast, for the righthand panels of 
this figure we have integrated the neutrino emission over the whole northern hemisphere and then rescaled the result to the full sphere, i.e.,

$$
\begin{aligned}
L_{v, 2}(t) & =2 r^{2} \int_{\text {hemisphere }} \mathrm{d} \Omega F_{v}(r, \theta, t) \\
& =4 \pi r^{2} \sum_{j=1}^{N_{\theta} / 2}\left(\cos \theta_{j-1}-\cos \theta_{j}\right) F_{v}\left(r, \theta_{j-\frac{1}{2}}, t\right),
\end{aligned}
$$

where the second line exploits axial symmetry and the sum is performed over the angular zones of one hemisphere. The former approach means that one assumes that an observer at a position near the polar axis receives the radiation emitted only from one pole-near lateral zone (in our treatment this means that the received radiation flux has the properties calculated in exactly one angular grid bin or angular "ray"), whereas the second evaluation implies the assumption that each unit of area on the hemisphere oriented towards the observer contributes to the observable luminosity with a weight defined by the (radial) flux calculated for the corresponding angular bin ("ray") of the polar grid in our ray-by-ray transport treatment. This assumption would be correct, of course, if a spherical source were radiating uniformly and isotropically in all directions, but in general the surface parts oriented with smaller angles to the observer direction contribute more strongly. For a sphere with a sharp radiating surface (in contrast to a radially extended neutrino-decoupling layer) that emits neutrinos with a (locally) isotropic intensity towards an observer at great distance on the axis of symmetry of the source, Eq. (3) would contain a factor $(2 \cos \theta)$ in the integrand ${ }^{4}$. If the intensity was a function of the latitudinal angle $\theta$ of the stellar grid, for example, this factor would enhance the relative contributions of surface parts that are closer to the observer. Because of limb darkening the importance of the observer-near regions of the radiating neutrinosphere is further enhanced.

Not solving the full multi-angle problem of neutrino transport in the $2 \mathrm{D}$ geometry but using our ray-by-ray transport approximation does not allow us to exactly compute the direction dependence of the neutrino intensity radiated from the nascent neutron star. The ray-by-ray treatment essentially implies the construction of a 1D transport solution in every lateral bin of the computational polar grid, assuming a spherically symmetric transport problem for the stellar conditions that are present in this angular bin. Therefore the ray-by-ray approach tends to underestimate the directional smearing of luminosity features that result from local and time-dependent emission increase in the semi-transparent accretion layer near the neutron star surface. Such features are, for example, caused by the SASI and convection modulated mass inflow and must be expected to be more prominent in the plots showing the measurable luminosity $L_{v, 1}$, because this quantity is the isotropic equivalent luminosity resulting from neutrino emission occurring in one angular grid bin (see lefthand panels of Fig. 6). In contrast, spatially localized effects will show up only with significant damping in the luminosity $L_{v, 2}$, where neutrinos coming from the whole hemisphere facing the observer are added up with equal weighting (righthand panels of Fig. 6). We consider these two evaluated cases as the extrema that define the theoretical limits of the true result. We suspect that $L_{\gamma, 1}$ yields the better approximation of the real situation, but we also provide $L_{v, 2}$ for a pessimistic estimate of the magnitude of the luminosity fluctuations. It is important to note

\footnotetext{
${ }_{4}$ The factor $\cos \theta$ accounts for the projection of the surface elements on the radiating sphere perpendicular to the line connecting source and observer.
}

that the total energy loss of the supernova core in neutrinos (or one sort of neutrinos), which is usually defined as neutrino luminosity and computed by integrating the neutrino flux over all observer directions, shows SASI induced variability only on a much reduced level (see the corresponding plots in the Marek \& Janka 2007 paper). This can be understood from the fact that the SASI fluctuations in the northern and southern hemispheres are phase shifted by roughly half a SASI period so that the emission maxima on one side fill the emission minima on the other. The combined signal is therefore much smoother than the neutrino flux that can be received by an observer in any chosen direction.

The modulations of the apparent luminosity due to SASI and convective variations of the neutron star accretion rate in the 2D simulations are superimposed on the general luminosity evolution that is characteristic of the employed progenitor star. These modulations add another feature on top of the effects that discriminate 1D from 2D results and models based on the use of the L\&S EoS from those with the H\&W EoS. The $15 M_{\odot}$ stellar model used in the present simulations shows a distinct drop in the mass accretion rate by roughly a factor of two when the steep density decline at the interface between the silicon shell and the oxygen-enriched Si layer falls through the stalled shock (see Appendices A and B of Buras et al. 2006b). This happens between $160 \mathrm{~ms}$ and $180 \mathrm{~ms}$ and leads to the pronounced decrease of the luminosities of all neutrinos and antineutrinos at around this time.

In Figs. 6 and 7 we see an effect already mentioned in Sect. 3.1, namely that the proto-neutron star in the simulations with the softer L\&S EoS is more compact and hotter and therefore radiates higher luminosities and significantly higher mean energies of all kinds of neutrinos and antineutrinos (the mean neutrino energies $\left\langle\epsilon_{v}\right\rangle$ are defined here as the ratio of the energy flux to the number flux). This holds for 1D as well as 2D models. Besides the quasi-periodic fluctuations, the $2 \mathrm{D}$ results exhibit the characteristic differences compared to the $1 \mathrm{D}$ case that were discussed before in much detail by Buras et al. (2006b): convective transport below the neutrinospheres leads to an accelerated loss of lepton number and energy, which manifests itself on the one hand in a slightly enhanced electron neutrino flux relative to the electron antineutrino flux, and on the other hand in significantly increased muon and tau neutrino luminosities compared to the $1 \mathrm{D}$ results. These differences are visible in both the polar luminosities, $L_{v_{i}, 1}$, as well as in the hemispherically integrated data, $L_{v_{i}, 2}$, of Fig. 6 . On the other hand, the convective neutron star is less compact than its $1 \mathrm{D}$ counterpart, for which reason the mean energies of the radiated neutrinos are slightly reduced in the 2D simulations (compare the upper two panels of Fig. 7 with the bottom ones). This phenomenon is particularly strong for the L\&S EoS, in which case the more compact neutron star structure seems to react more sensitively to the redistribution of lepton number and entropy associated with the convective activity in its interior.

An interesting effect is the crossing of the mean energies of electron antineutrinos and heavy-lepton neutrinos $\left(\left\langle\epsilon_{\bar{v}_{\mathrm{e}}}\right\rangle\right.$ becoming higher than $\left.\left\langle\epsilon_{v_{x}}\right\rangle\right)$ that occurs in five of the six panels of Fig. 7 at about $200 \mathrm{~ms}$ after bounce, but which cannot be observed until this post-bounce time for other energy moments; for example it is absent in the case of the rms energies of the energy spectra. These rms energies are ordered in the standard way at all times, with heavy-lepton neutrinos $v_{x}$ being more energetic on average than $\bar{v}_{\mathrm{e}}$, and the latter being more energetic than $v_{\mathrm{e}}:\left\langle\epsilon_{v_{\mathrm{e}}}\right\rangle_{\mathrm{rms}}<\left\langle\epsilon_{\bar{v}_{\mathrm{e}}}\right\rangle_{\mathrm{rms}}<\left\langle\epsilon_{v_{x}}\right\rangle_{\mathrm{rms}}$ (this was pointed out already by Marek \& Janka 2007). The energy crossing therefore is a consequence of a subtle difference in the spectral shape 
of $\bar{v}_{\mathrm{e}}$ and $v_{x}$. A close inspection of our results reveals that this effect is connected with the modification of the radiated neutrino fluxes in the accretion layer around a successively more compact, contracting proto-neutron star. In contrast to the mean energies at a radius of $400 \mathrm{~km}$, i.e. far outside of the neutrino source (see Fig. 7), the corresponding data at a fixed density of $10^{11} \mathrm{~g} \mathrm{~cm}^{-3}$ always follow the relation $\left\langle\epsilon_{\bar{v}_{\mathrm{e}}}\right\rangle \lesssim\left\langle\epsilon_{v_{x}}\right\rangle$. This holds for our $1 \mathrm{D}$ results and for the time-dependent unidirectional as well as (hemi)spherically averaged 2D results with both equations of state; only during short phases of a strong increase of the accretion rate can the mean energy of electron antineutrinos exceed the one of heavy-lepton neutrinos also at $\rho=10^{11} \mathrm{~g} \mathrm{~cm}^{-3}$. Similarly, accretion around the poles of the 2D models also causes the polar rms energies of $\bar{v}_{\mathrm{e}}$ and $v_{x}-$ measured outside of the accretion layer - to converge $\left(\left\langle\epsilon_{\bar{v}_{\mathrm{e}}}\right\rangle_{\mathrm{rms}} \lesssim\left\langle\epsilon_{v_{x}}\right\rangle_{\mathrm{rms}}\right)$, whereas the laterally averaged values are always well separated, in agreement with the hierarchy of the rms energies found in the corresponding 1D simulations. Moreover, the neutrino radiating "naked" neutron star in spherically symmetric models with artifically initiated explosions does not produce the inversion of the mean $\bar{v}_{\mathrm{e}}$ and $v_{x}$ energies, whereas the accreting neutron star in a corresponding nonexploding model does (see Fig. 2 in Janka et al. 2007).

All these findings point to the importance of accretion for an explanation of the phenomenon. Accretion modifies the density profile outside of the neutrinosphere and thus is responsible for a subtle change in the $\bar{v}_{\mathrm{e}}$ spectrum relative to the $v_{x}$ spectrum. Two effects seem to contribute. On the one hand, with the inclusion of $v_{x} \bar{v}_{x}$-pair production by nucleon-nucleon bremsstrahlung and by $v_{\mathrm{e}} \bar{v}_{\mathrm{e}}$ annihilation in state-of-the-art supernova models, the energysphere of heavy-lepton neutrinos is shifted to lower temperatures than in older simulations where these processes were ignored. The heavy-lepton neutrinos that leave their average neutrino-energysphere also experience a "spectral filter effect" in being down-graded in energy space by neutrino-nucleon and neutrino-electron collisions when diffusing through the cooler, outer layers of the neutron star (modern multi-energy transport calculations follow the corresponding spectral changes with great accuracy). For both reasons the spectra of $v_{x}$ and $\bar{v}_{\mathrm{e}}$ are now much more similar than in the older models (for a detailed discussion, see Raffelt 2001; Keil et al. 2003). On the other hand, electron neutrinos and antineutrinos, which are emitted in rapid charged-current reactions of electrons and positrons with protons and neutrons, are more copiously produced in the hot accretion layer than muon and tau neutrinos. This leads to an enhancement of the $\bar{v}_{\mathrm{e}}$ flux at medium and high energies $(\gtrsim 10 \mathrm{MeV})$ compared to the $v_{x}$ flux at these energies. In combination, these effects can raise the average energy of electron antineutrinos radiated from an accreting neutron star above that of heavy-lepton neutrinos.

The described relative spectral changes become stronger at later times after core bounce so that a crossing of $\left\langle\epsilon_{\bar{v}_{\mathrm{e}}}\right\rangle$ and $\left\langle\epsilon_{v_{x}}\right\rangle$ occurs. As the post-bounce time advances, the average energies of muon and tau neutrinos grow slowly because the neutrinospheric temperature increases in the shrinking neutron star. This increase is stronger for the soft L\&S EoS than for the stiff H\&W EoS (see Fig. 7). In contrast, the $v_{\mathrm{e}}$ and $\bar{v}_{\mathrm{e}}$ created in the semitransparent accretion layer escape with mean energies that rise relatively faster, because accretion onto a more compact proto-neutron star releases more gravitational binding energy. This leads to more extreme heating of the infalling and compressed gas and more abundant production of $v_{\mathrm{e}}$ and $\bar{v}_{\mathrm{e}}$ with higher energies. This reasoning also explains why the polar-near data in Fig. 7 show the effect more clearly than the hemispherically averaged results: the SASI sloshing motions of the shock along the direction of the polar grid axis with alternating periods of shock inflation and contraction lead to successive phases of gas expansion and mass accumulation on the one hand, and gas compression on the other, in particular around the north and south poles of the neutron star (compare Figs. 3 and 6).

At first sight this argument seems in conflict with the observation that the accretion funnels in the sequence of snapshots of Fig. 3, in particular the most prominent and persistent ones that carry most mass, reach down to the neutron star typically at intermediate latitudinal angles, i.e., around the equator; still the fluctuations of the neutrino emission are highest close to the poles. We understand this as a consequence of the following two facts. Firstly, because of the extremely large infall velocities only little neutrino loss occurs within the downdrafts. Instead, most of the binding energy is radiated when the accreted gas spreads around the neutron star and begins to settle onto the neutron star surface (for a discussion of this aspect, see also Scheck et al. 2006). Secondly, due to the SASI sloshing of the whole accretion layer, much of the gas accreted through the shock is redirected alternatingly towards the north pole or the south pole. There the gas is decelerated near the grid (and sloshing) axis and deflected so that its duration of stay is longest in the polar regions. The gas that is sucked to a pole at an enhanced rate during the SASI halfcycle of shock expansion is compressed there during the next half-cycle of shock contraction. This is the reason why most of the losses of accretion energy happen around the poles. Future three-dimensional (3D) simulations with neutrino transport will have to show whether this behavior is a consequence of the assumption of axial symmetry or whether a similar effect can also be found in connection with the SASI in three dimensions.

Concerning the possibility of measuring the SASI (and convection) induced luminosity variations by the detection of neutrinos from a future Galactic supernova it is important to note that the strong compressional heating of accreted gas during the SASI half-cycles with shock contraction does not only boost the neutrino emission at the poles but also leads to the production of more energetic neutrinos. Therefore the variations of the neutrino luminosities correlate and are in phase with modulations of the mean neutrino energies (see in particular the polar data in Figs. 6 and 7). While (for the polar emission) the luminosity differences between maxima and minima can be several $10 \%$ up to about $50 \%$ of the values in low-emission episodes and the mean energies show variations up to roughly $1 \mathrm{MeV}$ or $10 \%$, the observationally relevant energy moments (approximately scaling like $L\langle\epsilon\rangle^{2}$ or $L\langle\epsilon\rangle$, depending on the neutrino detector) fluctuate with an amplitude of typically $\sim 50 \%$ and extrema up to nearly $100 \%$ of the values in the minima. The fluctuations are similarly strong for $v_{\mathrm{e}}$ and $\bar{v}_{\mathrm{e}}$, which are both abundantly created in the accretion layer, and somewhat reduced for heavy-lepton neutrinos, whose emission is more indirectly affected by the perturbations of their decoupling region near the neutron star surface, e.g. through the surface gravity modes caused by the SASI activity and through changes in the density structure and optical depth associated with the mass motions in the SASI region.

In Fig. 8 we provide the Fourier spectra of the neutrino luminosities displayed in Fig. 6; the lefthand panels belong to the polar luminosities, the righthand panels to the hemispherically averaged ones. The 2D simulations exhibit a broad peak between about $20 \mathrm{~Hz}$ and roughly $400 \mathrm{~Hz}$ with a clear power excess compared to $1 \mathrm{D}$ results. Instead of the expected monotonic decline the Fourier transforms of the 1D luminosities possess a flat shoulder at frequencies between $\sim 50 \mathrm{~Hz}$ and $\sim 100 \mathrm{~Hz}$. This feature is a consequence of low-amplitude numerical noise 

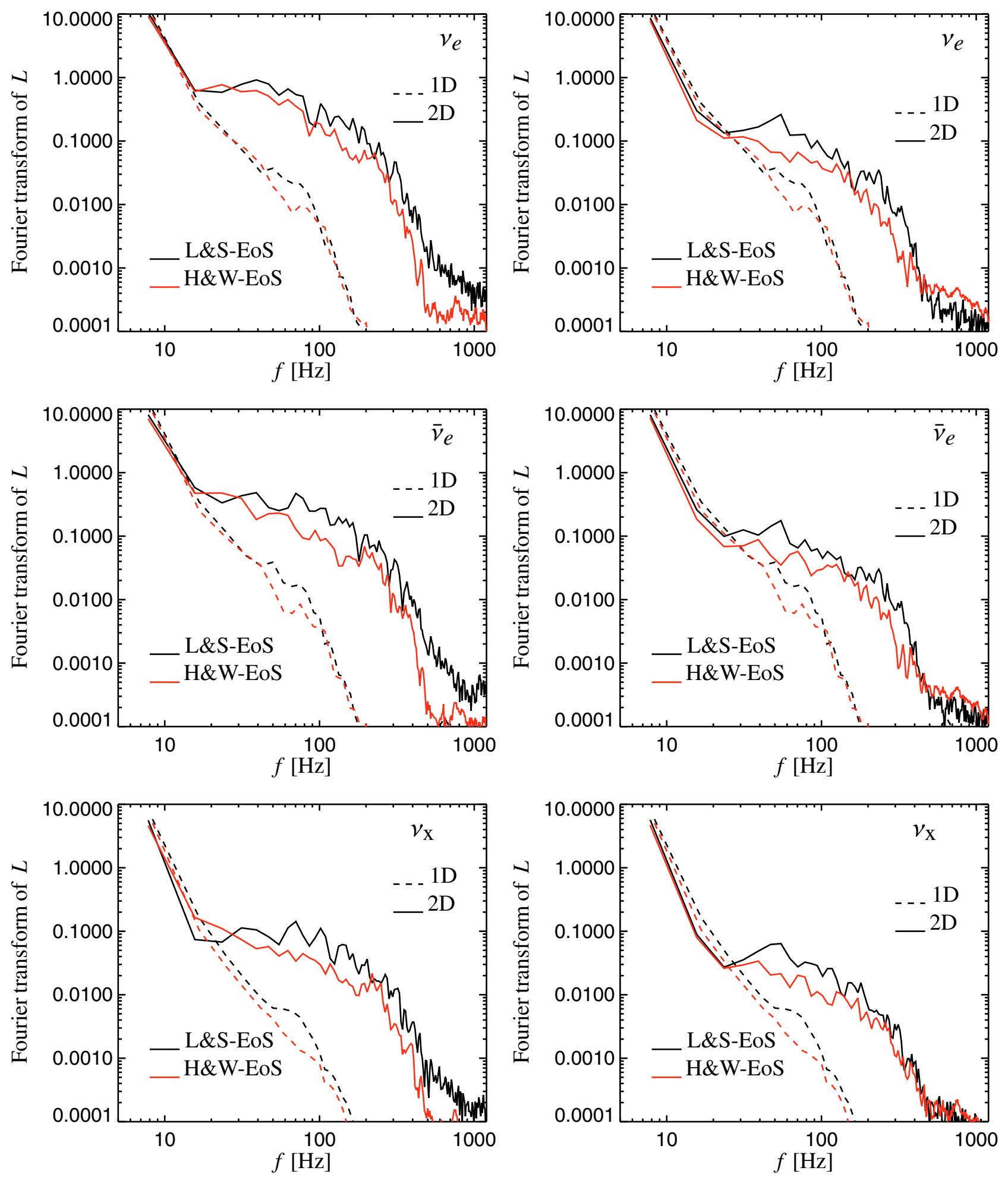

Fig. 8. Fourier amplitudes of the time-dependent neutrino luminosities shown in Fig. 6 (the left/right column there corresponds to the left/right column in the present figure). In order to reduce the level of background noise, the evaluation was performed for post-bounce times $t>100$ ms, for which the SASI modulation is clearly visible in the neutrino luminosities, and the signals were split in 17 overlapping segments of 128 ms, advanced in steps of $10 \mathrm{~ms}$. The Fourier spectra of the $1 \mathrm{D}$ models show a weak contribution from low-amplitude numerical fluctuations of the transport results.

produced by the transport scheme, which is so small that it is physically insignificant for the simulations. As expected from the time evolution of the shock radii and corresponding luminosity variations, which both are larger in the case of the L\&S run, the Fourier spectra of the $2 \mathrm{D}$ simulation with the
L\&S EoS dominate the result with the H\&W EoS by a factor of $2-5$ for all kinds of neutrinos. The flat local maxima in the $\sim 25-$ $80 \mathrm{~Hz}$ range, whose location roughly agrees with the peak of the Fourier amplitude of the $\ell=1$ shock oscillation mode (Fig. 5) and also shows the tendency of slightly higher frequencies for 
the L\&S case, are superimposed by a high-frequency component that originates from the faster convective modulations of the accretion flow and neutrino emission.

\subsection{Gravitational waves}

Nonradial mass motions in the three regions of hydrodynamic instabilities as well as anisotropic neutrino emission are sources of gravitational-wave signals from stellar core-collapse events (e.g., Müller et al. 2004 and references therein). We will analyze our data for EoS-specific properties of these signals now.

The (quadrupole) gravitational-wave amplitudes, energy spectra, and spectrograms resulting from anisotropic mass motion can be computed for axisymmetric models as described in Müller \& Janka (1997; Eqs. (10)-(12)), using the Einstein quadrupole formula in the numerically convenient form derived by Blanchet et al. (1990), and by standard FFT techniques. Assuming an observer that is located at an angle $\theta$ with respect to the symmetry axis of the source, the dimensionless gravitational wave amplitude $h(t)$ is related to the quadrupole wave amplitude $A_{20}^{E 2}$ (measured in units of $\mathrm{cm}$ ), the lowest-order nonvanishing term of a multipole expansion of the radiation field into pure-spin tensor harmonics (see Eq. (9) of Müller 1997), according to

$h=\frac{1}{8} \sqrt{\frac{15}{\pi}} \sin ^{2} \theta \frac{A_{20}^{E 2}}{R}$,

where $R$ is the distance to the source. In the following we will always assume $\sin ^{2} \theta=1$, i.e., the observer is positioned in the equatorial plane of the polar grid. The wave amplitudes due to the anisotropic neutrino emission are obtained with the formalism given in Müller \& Janka (1997, Eqs. (28)-(31)), which is based on the work of Epstein (1978). According to these references, the dimensionless gravitational-wave amplitude for the radiated energy in neutrinos can be written as convolution of the time-dependent neutrino luminosity $L_{v}(t)$ of the supernova core and the emission anisotropy $\alpha_{v}(t)$,

$h_{v}^{\mathrm{TT}}=\frac{2 G}{c^{4} R} \int_{0}^{t} \mathrm{~d} t^{\prime} L_{v}\left(t^{\prime}\right) \alpha_{v}\left(t^{\prime}\right)$

(the quadrupole wave amplitude $A_{20}^{E 2}$ for neutrinos then results from inversion of Eq. (5)), with

$\alpha_{\nu}(t) \equiv \frac{1}{L_{v}(t)} \int_{4 \pi} \mathrm{d} \Omega \Psi(\theta, \phi) \frac{\mathrm{d} L_{\nu}(\boldsymbol{\Omega}, t)}{\mathrm{d} \Omega}$,

where $\mathrm{d} L_{v}(\boldsymbol{\Omega}, t) / \mathrm{d} \Omega$ is the neutrino energy radiated at time $t$ per unit of time and per unit of solid angle into direction $\mathrm{d} \Omega$, and $\mathrm{d} \Omega=-\mathrm{d} \cos \theta \mathrm{d} \phi$ defines the solid angle element in the polar coordinate system of the source. The angle-dependent function $\Psi(\theta, \phi)$ is given in Eq. (27) of Müller \& Janka (1997). For the case of an axially symmetric source, Kotake et al. (2007) derived a compact expression (see their Eq. (8) and note that our $\Psi$, following the notation of Müller \& Janka 1997, is their $\Phi)$, which is visualized as a function of the polar angle $\theta$ in their Fig. 1. For the further discussion it is important to note that $\Psi$ has positive values in the polar cap regions between $\theta=0$ and $\theta=60^{\circ}$ and between $\theta=120^{\circ}$ and $\theta=180^{\circ}$, but adopts negative values in the equatorial belt for $60^{\circ}<\theta<120^{\circ}$. The combined polar caps and the equatorial belt have equally large surface areas.

The quadrupole wave amplitudes $A_{20}^{E 2}$ of our two 2D models and the corresponding Fourier spectra are shown in Fig. 9, in the upper panels for the signals from aspherical matter flow, and in the lower panels for those from anisotropic neutrino emission.
The matter signal consists of a superposition of quasiperiodic variations on timescales ranging from a few to several ten milliseconds. Two phases of enhanced activity can be distinguished. The first one occurs within tens of milliseconds after core bounce and is followed by a more quiescent episode before a long-lasting period with an overall trend in growing amplitudes begins. The transient, early phase of strong gravitational-wave emission is a consequence of the prompt post-shock convection (cf. Sect. 3.1) and exhibits larger amplitudes, slightly higher frequencies (spectral peak at about $100 \mathrm{~Hz}$ instead of $\sim 70 \mathrm{~Hz}$ ), and more power in our simulation with the stiffer $\mathrm{H} \& \mathrm{~W}$ EoS (see Fig. 10). The reason for these differences is the fact that the region of prompt convection involves more mass and extends to larger radii in the $\mathrm{H} \& \mathrm{~W}$ case (Figs. 1 and 2), where also the growth rates of the instability are higher and the mass motions become more violent and faster. The prominent peaks of the Fourier spectra in Fig. 10 correspond to a clearly dominant frequency that can be identified in the wave train during the first $\sim 80 \mathrm{~ms}$ after bounce.

The later phase of intense gravitational-wave production is associated with the development of convective overturn and large-amplitude SASI oscillations in the post-shock layer as well as mass flows and convective activity in the proto-neutron star. Accordingly, the wave amplitudes at $t \gtrsim 100 \mathrm{~ms}$ show variability with different frequencies. It is interesting to note that the enveloping curves of the wave amplitudes at this time follow roughly the evolution of the maximum shock radii in Fig. 4. This means that more active phases and stronger gravitationalwave emission correlate with larger shock radii. The nonradial gas motions in the post-shock region contribute mostly to the power radiated at frequencies up to about $200 \mathrm{~Hz}$ in accordance with the SASI Fourier spectra shown in Fig. 5. The main peak of the matter gravitational-wave spectrum at $300-600 \mathrm{~Hz}$ $(\mathrm{H} \& \mathrm{~W}$ EoS) and $600-800 \mathrm{~Hz}(\mathrm{~L} \& \mathrm{~S}$ EoS) as well as most of the lower "background" above $f \sim 200 \mathrm{~Hz}$, however, originates from changes in the mass-quadrupole moment in deeper regions, namely at densities between $10^{11} \mathrm{~g} \mathrm{~cm}^{-3}$ and $10^{13} \mathrm{~g} \mathrm{~cm}^{-3}$, where more mass is involved than in the post-shock layer. The main source of this signal are gravity modes and gas flows instigated by the violent impact of accretion funnels in the surface layers of the nascent neutron star, which perturbs regions at densities up to $\rho \sim 10^{12} \mathrm{~g} \mathrm{~cm}^{-3}$. A substantial part of the emission also comes from the convective zone inside the neutron star at $\rho>10^{12} \mathrm{~g} \mathrm{~cm}^{-3}$. The importance of the interaction of the SASI and accretion flows with the proto-neutron star surface can be concluded from a closer inspection of the upper lefthand panel of Fig. 9 in comparison with Fig. 1. The gravitational-wave amplitudes of the L\&S run at $t>100 \mathrm{~ms}$ p.b. are significantly larger than those of the H\&W model until near the end of our simulations. Only then the amplifying SASI and accretion activity begins to affect shells at $\rho \sim 10^{12} \mathrm{~g} \mathrm{~cm}^{-3}$ also in the $\mathrm{H} \& \mathrm{~W}$ case, while earlier the involved regions in this model possess clearly lower densities.

The maximum values of the dimensionless gravitationalwave strain $h(t)$ for the matter signal are of the order of several $10^{-22}$ for a source at a distance of $10 \mathrm{kpc}$, and the dominant peak may be marginally observable with LIGO I in the case of a Galactic supernova (for a detailed discussion of measurement aspects, see Müller et al. 2004). While the spectral peak is slightly higher if the nuclear EoS is soft and the proto-neutron star more compact, the stiffer $\mathrm{H} \& \mathrm{~W}$ EoS leads to somewhat lower peak frequencies and thus to a shifting of the main emission closer to the region of highest sensitivity of the LIGO instrument. It is 

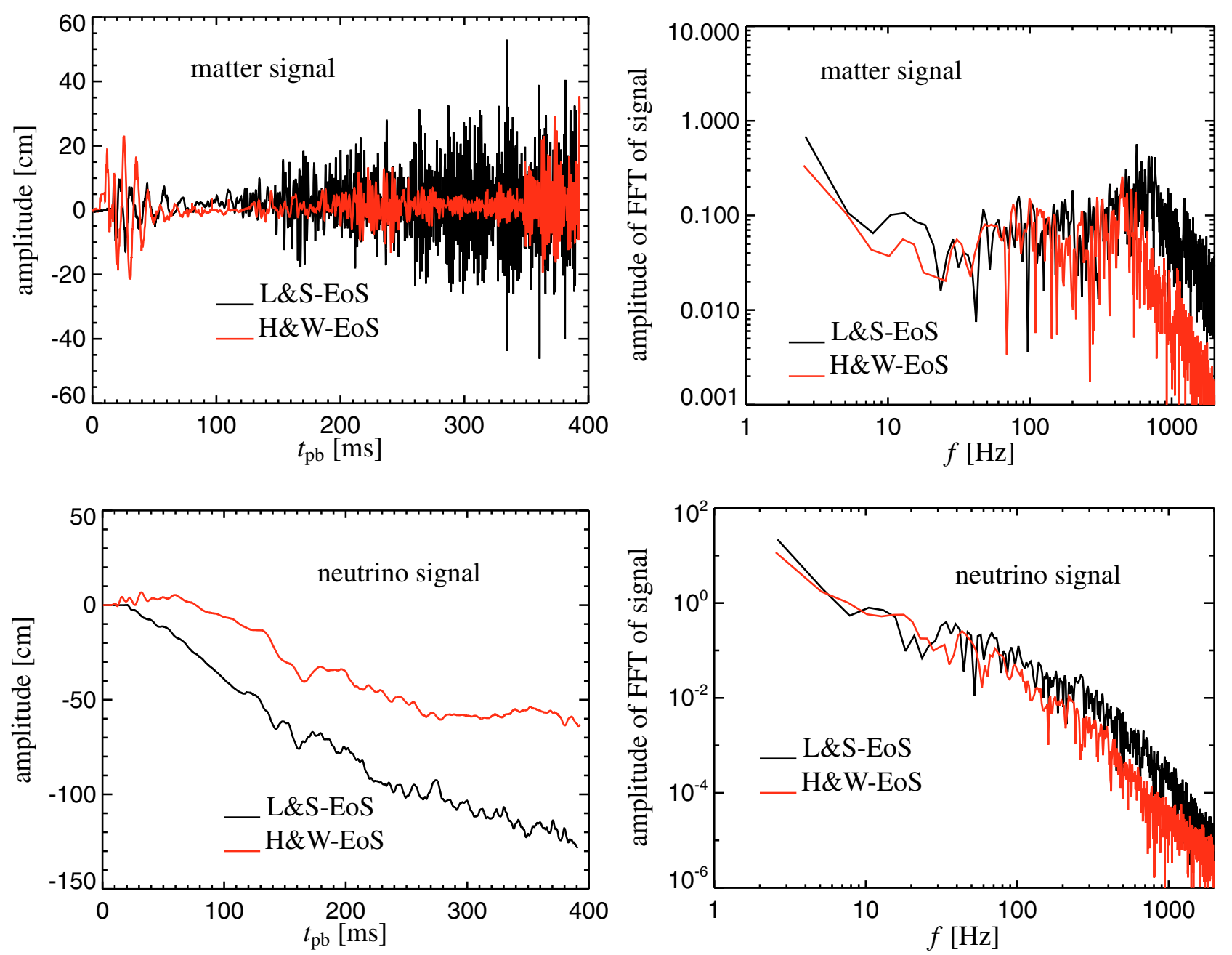

Fig. 9. Gravitational-wave quadrupole amplitudes $A_{20}^{E 2}$ as functions of post-bounce time (left) and corresponding Fourier spectra (right) associated with mass motions (top) and anisotropic neutrino $\left(v_{\mathrm{e}}+\bar{v}_{\mathrm{e}}\right.$ plus all heavy lepton neutrinos and antineutrinos) emission (bottom) for a distant observer in the equatorial plane of the axisymmetric source. Note that despite the trend toward a prolate shock deformation at late post-bounce times (see Fig. 3 and the lefthand panels of Fig. 5) the neutrino amplitude develops negative values, suggesting that the signal is not determined by a dominant polar neutrino emission in this case. The prominent peaks of the matter signals at Fourier frequencies around $500 \mathrm{~Hz}$ and $700 \mathrm{~Hz}$, respectively, are caused by mass motions in the proto-neutron star surface at densities between $10^{11} \mathrm{~g} \mathrm{~cm}^{-3}$ and $10^{13} \mathrm{~g} \mathrm{~cm}^{-3}$.

therefore not clear that a soft EoS is more favorable for a measurement of such gravitational-wave signals.

Significantly larger amplitudes are generated by the anisotropic neutrino emission, however most power of this component of the gravitational-wave signal comes at frequencies below $100 \mathrm{~Hz}$ (Fig. 9). The lower lefthand panel of Fig. 9 shows negative amplitudes with the continuous trend in growing absolute values and superimposed high-frequency modulations for both $2 \mathrm{D}$ simulations discussed in this paper. The negative values indicate a time-dependent quadrupole moment that is determined by an excess of neutrino emission from regions near the equatorial plane, a fact that can be concluded easily from the latitudinal variation of the function $\Psi(\theta)$ in the angular integral of the anisotropy parameter $\alpha_{v}(t)$ of Eq. (7). This function becomes negative in the equatorial belt between $60^{\circ}$ and $120^{\circ}$, see Fig. 1 of Kotake et al. (2007).

A continuous decrease of the neutrino gravitational-wave amplitude to negative values is no common feature of all 2D simulations. This behavior depends strongly on the particular emission asymmetry that develops within the nascent neutron star and in its accretion layer. Müller et al. (2004) found long-period variations between positive and negative values, for example, for an explosion simulation of an 11.2 $M_{\odot}$ star (cf. their Fig. 4) and for a 2D neutrino-cooling simulation of a convective proto-neutron star (cf. their Fig. 5), and they obtained a positive gravitationalwave amplitude with an overall trend in growth and only shorter periods of decrease for the neutrino emission of a centrifugally flattened, accreting neutron star at the center of a collapsing, rotating $15 M_{\odot}$ supernova progenitor (cf. their Fig. 3).

This diversity of possible behaviors is in conflict with results obtained by Kotake et al. (2007), who reported almost monotonically rising, positive wave amplitudes for all of their 2D simulations of SASI unstable accretion shocks in collapsing supernova cores. They explained their finding by the particular properties of the SASI and the corresponding post-shock accretion flow in models that are constrained by the assumption of axial symmetry. In this case the SASI oscillations must occur along the polar grid axis, and Kotake et al. (2007) argued that the influence of this on the accretion flow makes the neutrino emission biased towards the regions around the symmetry axis (see also Sect. 3.2), thus leading to positive and growing wave amplitudes.

Why do we come to different conclusions about the neutrino emission asymmetry than Kotake et al. (2007) in spite of our apparent agreement about the role of the SASI for the $v_{\mathrm{e}}$ and 


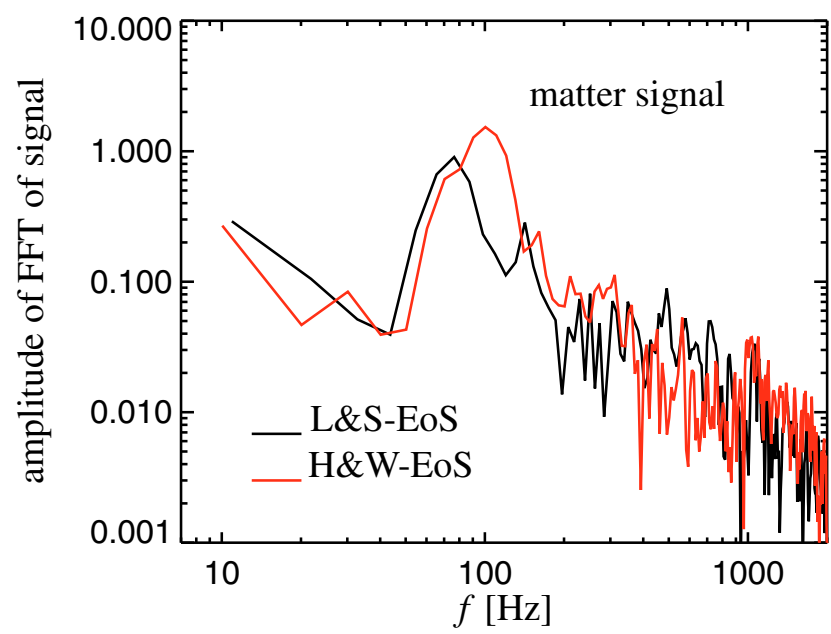

Fig. 10. Fourier spectra of the gravitational wave quadrupole amplitudes of the mass motions caused by prompt post-shock convection during the first $100 \mathrm{~ms}$ after core bounce (see upper lefthand panel of Fig. 9).

$\bar{v}_{\mathrm{e}}$ production in the accretion layer? In the first place one must note that the modeling approach taken by Kotake et al. (2007) is fundamentally different from ours. Besides many other differences, they, for example, did not simulate the collapse of a stellar progenitor model but started from a steady-state solution of an accretion shock with a mass accretion rate of $1 M_{\odot} \mathrm{s}^{-1}$; they excised the neutron star interior at a constant radius chosen as the inner boundary of the computational grid, where they assumed a fixed density of $10^{11} \mathrm{~g} \mathrm{~cm}^{-3}$; and they kept the fairly high mass accretion rate and the accretor mass constant during all of the simulation (time-independent boundary conditions at the outer grid boundary), using a spherically symmetric Newtonian gravitational potential and ignoring self-gravity of the matter in the accretion flow. Moreover and very important in the context of the present discussion of gravitational-wave signals associated with anisotropic neutrino emission, they did not solve the energy-dependent, multi-flavor neutrino transport problem but employed a light-bulb description with prescribed, timeindependent neutrino luminosities and spectra from the nascent neutron star and local source rates for lepton number and energy due to neutrino reactions in the accretion flow.

Based on the possibilities available to us for comparison we cannot make a reasonable judgement whether any, and if so, which of these simplifications are causal for the discrepant gravitational-wave results obtained by us and by Kotake et al. (2007). However, we strongly suspect that the approach of the latter work is too simplistic for reliably estimating the emission asymmetry of neutrinos that is responsible for the gravitationalwave generation. This conclusion is supported by a close analysis of our neutrino transport data and the insight they yield into the formation of the neutrino emission asymmetry. Figure 11 provides the corresponding information for our 2D simulation with the L\&S EoS.

In the upper lefthand panel of Fig. 11 we display the time evolution of the neutrino gravitational-wave amplitude for this model. Different from Fig. 9 we have performed the evaluation now not only at a radius of $400 \mathrm{~km}$, where the neutrino luminosity has reached its asymptotic level, but also at the (timedependent) radius where the density has a value of $10^{11} \mathrm{~g} \mathrm{~cm}^{-3}$, i.e. in the vicinity of the electron neutrinosphere and thus below the accretion layer where neutrino cooling still enhances the outgoing $v_{\mathrm{e}}$ and $\bar{v}_{\mathrm{e}}$ fluxes. The upper righthand panel of Fig. 11 contains the corresponding parameters $\alpha_{v}(t)$ of the neutrino emission anisotropy, which exhibit rapid variations between positive and negative values on timescales of milliseconds to $\gtrsim 10 \mathrm{~ms}$ and growing amplitudes at later times. Both upper panels show an even clearer long-time trend to negative gravitationalwave amplitudes for the neutrino luminosity leaving the neutrinospheric layer: $\alpha_{v}(t)$ measured at $10^{11} \mathrm{~g} \mathrm{~cm}^{-3}$ attains positive values only during the very short time intervals when the highfrequency modulations reach their largest amplitudes, while this parameter at a radius of $400 \mathrm{~km}$ enters the positive side for significantly more numerous and longer time intervals, although $\int_{0}^{t} \mathrm{~d} t^{\prime} \alpha_{v}\left(t^{\prime}\right)<0$ for all times at both locations. Correspondingly, the gravitational-wave amplitude at $\rho=10^{11} \mathrm{~g} \mathrm{~cm}^{-3}$ is even more negative than the one at $r=400 \mathrm{~km}$, and their difference (green line in the upper lefthand panel of Fig. 11) suggests that the amplitude associated with the neutrino emission in the accretion layer is positive and reduces the neutrinospheric emission asymmetry. This seems to mean that the final emission anisotropy of the neutrinos is determined already by the flux that leaks out from the neutrinospheric region and in which the equatorial region has obtained a slight flux excess. Below we will see, however, that this interpretation is not completely correct and the true situation is more complex. A comparion of the different lines in the upper lefthand panel of Fig. 11 also reveals that the fluctuations of the radiated neutrino gravitational-wave signal with a period of 10-20 ms (frequency $\sim 50-100 \mathrm{~Hz}$ ) originate from the accretion layer and are therefore connected to the SASI and convective activity in the post-shock region and its influence on the neutrino-cooling zone as described in Sect. 3.2.

\subsubsection{Neutrino emission anisotropy}

In order to obtain deeper insight into the origin of the neutrino emission anisotropy, we have calculated the cumulative loss of energy in neutrinos (i.e., the time- and surface-integrated neutrino flux) once for the polar cap regions and another time for the equatorial belt, again at $r=400 \mathrm{~km}$ and at $\rho=10^{11} \mathrm{~g} \mathrm{~cm}^{-3}$. The results are shown in the lower lefthand panel of Fig. 11. The small differences $E_{\mathrm{e}}-E_{\mathrm{p}}$ between equatorial and polar neutrino energy losses, scaled by a factor of 10 , are plotted with dash-dotted lines. Again we see that at large distances as well as near the neutrinospheres the equatorial emission exceeds slightly the polar one during the whole evolution after $t \sim 40 \mathrm{~ms}$ post bounce, and this excess is reduced by the neutrino production that occurs in the cooling layer outside of a density of $10^{11} \mathrm{~g} \mathrm{~cm}^{-3}$. Separating the analysis for the different neutrino kinds, it becomes obvious that only the $v_{\mathrm{e}}$ and $\bar{v}_{\mathrm{e}}$ luminosities grow in the cooling layer above the neutrinospheres, and that these neutrinos are more abundantly produced in the polar regions where the loss of accretion energy mainly occurs, as discussed in Sect. 3.2. For electron neutrinos the emission in the accretion layer near the poles is so strong that their polar energy loss at large distances can become bigger than the equatorial one. In contrast, the flux of heavy-lepton neutrinos, which are radiated from the neutrinospheric region with a persistent surplus of the equatorial energy loss, gets a bit reduced in the accretion and postshock layers between $\rho=10^{11} \mathrm{~g} \mathrm{~cm}^{-3}$ and $r=400 \mathrm{~km}$, whereby their equatorial emission excess grows to roughly twice the value near the neutrinospheres. Their asymmetry determines the final emission asymmetry, because they contribute the major part $(\sim 56 \%)$ of the total neutrino energy loss (in fact, the pole-equator asymmetries of $v_{\mathrm{e}}$ and $\bar{\nu}_{\mathrm{e}}$ at large distances have opposite signs and nearly cancel each other). These findings are 

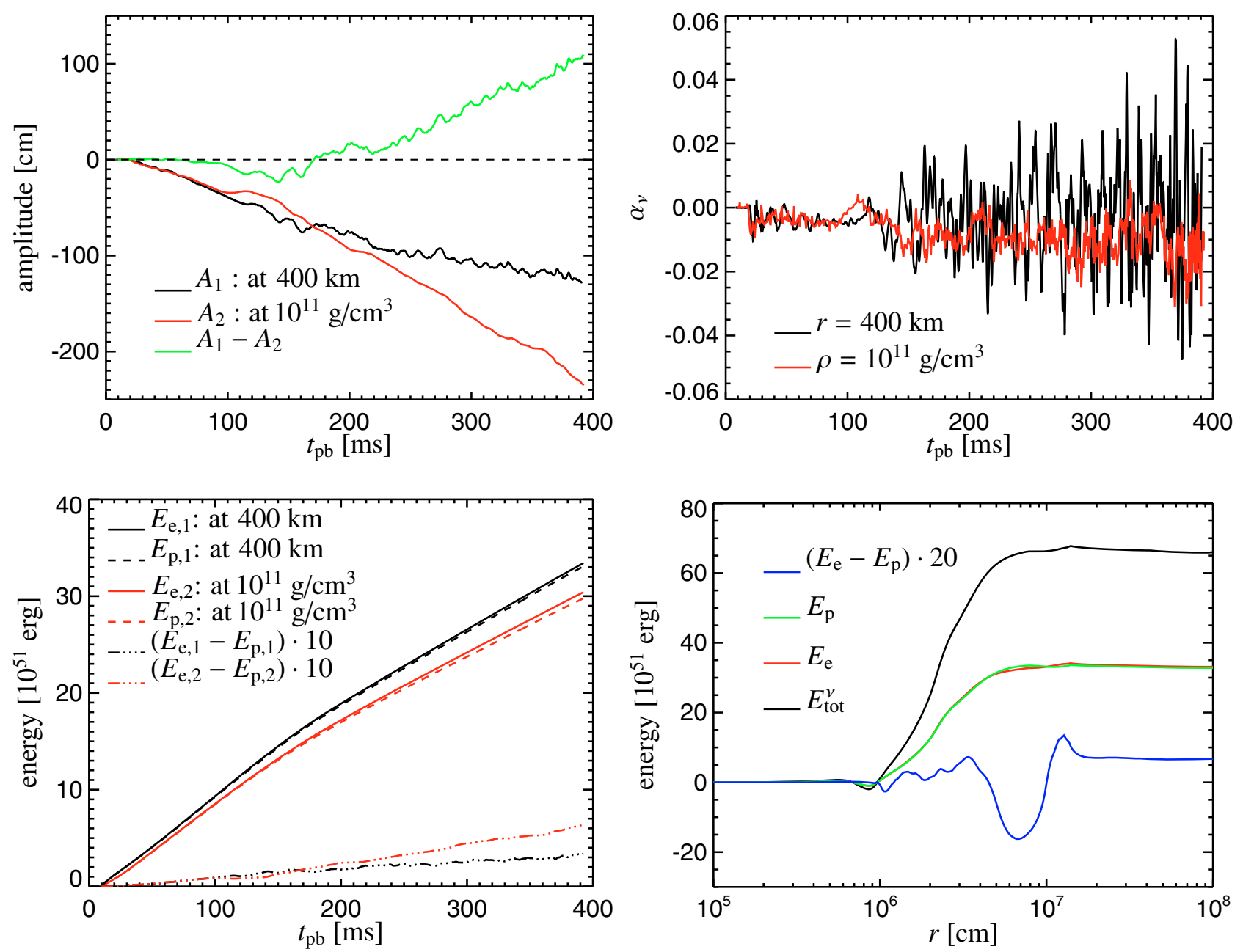

Fig. 11. Quantities that are relevant for the gravitational-wave signal associated with the anisotropic neutrino emission in our 2D model using the L\&S EoS. Top left: gravitational-wave amplitudes as functions of time after core bounce. The black curve was obtained by considering the neutrino radiation at a large distance from the source (identical with the corresponding line in the lower lefthand panel of Fig. 9, where, as in Figs. 6 and 7, the evaluation was performed in the laboratory frame at a radius of $400 \mathrm{~km}$ ). The red line shows the wave amplitude computed from the neutrino luminosities that leave the neutrinospheric region (rather, a sphere where the density is $10^{11} \mathrm{~g} \mathrm{~cm}^{-3}$ ). The green line displays the difference between the black and red curves. Top right: anisotropy parameter $\alpha_{v}$ of the neutrino emission as function of post-bounce time. The color coding of the curves is the same as in the previous plot. Bottom left: energy radiated in all neutrinos through the "polar cap regions" $\left(E_{\mathrm{p}}\right)$ and through the "equatorial belt" ( $E_{\mathrm{e}}$; see text for details), and the difference in these results (scaled by a factor of 10), integrated from the beginning of the $2 \mathrm{D}$ simulation shortly after bounce until the time $t_{\mathrm{pb}}$ on the horizontal axis. As in the two panels before, the color coding of the curves corresponds to the two different locations where this analysis was performed. Bottom right: total energy radiated in neutrinos through the "polar cap regions" $\left(E_{\mathrm{p}}\right)$, through the "equatorial belt" $\left(E_{\mathrm{e}}\right)$, difference in both scaled by a factor of 20 , and sum of both $\left(E_{\mathrm{tot}}^{v}\right)$ as functions of radius. The time integration was performed from the beginning of the $2 \mathrm{D}$ simulation shortly after bounce until the end of the model run at about $400 \mathrm{~ms}$ after bounce.

in clear contradiction to the results obtained by Kotake et al. (2007), who reported that the neutrino gravitational-wave signals in their models were dominated by the $v_{\mathrm{e}}$ emission and the other neutrinos did only contribute very small amplitudes with the same signs (see their Fig. 3).

What is the reason that slightly $(2-3 \%)$ more energy is radiated from the neutrinospheric layer near the equator than near the poles? We find that the emergence of this asymmetry coincides with the development of the stable convective shell inside the neutron star. This might suggest that convective transport near the equatorial plane is slightly more effective than in the polar regions. We have no satisfactory explanation for this phenomenon, because one would expect convection to operate similarly in all directions and not to produce a global asymmetry on angular scales much larger than the size of the (nonstationary) convective cells. This, however, is a statement that is correct in three dimensions but might not hold for convection in the $2 \mathrm{D}$ environment of our simulations. Here convective plumes and downflows are toroidal features because of the axial symmetry, and the polar grid has a preferred direction along the polar axis. We suspect - without having performed a detailed analysis - that the operation of convective overturn with cells of such a toroidal structure near and along the polar axis might be slightly different from the in- and outflows near the equatorial plane with fluid motions perpendicular to the axis. If this were indeed the origin of the neutrino emission asymmetry, it would imply a serious warning that the computation of such anisotropies for estimating gravitational-wave signals (and also pulsar kicks based on the recoil associated with anisotropic neutrino emission) requires 3D modeling, because in $2 \mathrm{D}$ the discussed effects may be just a consequence of the symmetry constraints.

But is this fishy directional asymmetry really responsible for our prediction of the neutrino gravitational-wave signal? Fortunately, it is not! To see this, we have evaluated our results 
in yet another way. In the lower righthand panel of Fig. 11 we display the cumulative - i.e. time-integrated from the beginning until the end of our 2D simulation - neutrino energy loss (again summed up for neutrinos and antineutrinos of all flavors) as a function of radius for the polar, equatorial, and total emission. The fourth (blue) curve represents the difference $\left(E_{\mathrm{e}}-E_{\mathrm{p}}\right)$ between equatorial and polar terms, scaled by a factor of 20 . Consistent with what we discussed above there is an excess of the equatorial losses until about $45 \mathrm{~km}$. This radius encompasses well the convection zone inside of the nascent neutron star during the whole post-bounce evolution of the 2D model with L\&S EoS (Fig. 1) and is larger than the location where $\rho=10^{11} \mathrm{~g} \mathrm{~cm}^{-3}$ for post-bounce times $t \gtrsim 170 \mathrm{~ms}$ (lefthand panel of Fig. 4). At greater radii when one enters the accretion and SASI layers, however, the polar energy loss first dominates before the equatorial one wins again at $r \gtrsim 100 \mathrm{~km}$. After a local maximum at a radius slightly larger than $100 \mathrm{~km}$ the equator-topole asymmetry settles to its final value. This radial variation is found to be qualitatively very similar for all kinds of neutrinos, although with different values of the local maxima and minima and of the final, asymptotic level at large radii.

From the lower righthand panel of Fig. 11 we learn that the emission anisotropy carried by the outgoing neutrinos is not imposed near the neutrinospheres and preserved outside, but it is completely determined by the accretion region behind the supernova shock and the SASI and convective inhomogeneities in this region. The final emission anisotropy just by chance gets close to the value near the neutrinospheres. Moreover, the separate analysis for the different kinds of neutrinos reveals that it is the muon and tau neutrinos that govern the final anisotropy of the radiated energy flux. In the two models presented here the energy carried away by these neutrinos in the equatorial belt is slightly higher than the one escaping from the polar cap areas. This as well as the total asymmetry, however, is likely to be a time- and model-dependent result and to depend strongly on the detailed structure of the inhomogeneities that evolve behind the deformed accretion shock. It could also depend on other model aspects such as the value of the mass accretion rate, the direction-dependent stagnation radius of the shock, or the relative sizes of the fluxes of the different neutrinos. Such conjectures are not only supported by the $\mathrm{H} \& \mathrm{~W}$ model in comparison to the L\&S run: the neutrino gravitational-wave amplitude of the latter model exhibits a significantly clearer trend in a monotonic decrease to negative values, while in the $\mathrm{H} \& \mathrm{~W}$ case one can see long phases where the amplitude or its time derivative become positive (Fig. 9). These conjectures are also in agreement with the results of other models described in the Müller et al. (2004) paper, and, last but not least, they are suggested by the fact that the quantity $E_{\mathrm{e}}-E_{\mathrm{p}}$ in the lower righthand panel of Fig. 11 is a quantity with large radial variations in both positive and negative directions. It is rather unlikely that such a complex radial behavior can be generic for models in two dimensions so that in all cases and at all times the same effects are recovered that ultimately determine the emission asymmetry in our models, namely (i) a delicate almost-cancellation of the combined asymmetry of $v_{\mathrm{e}}$ and $\bar{v}_{\mathrm{e}}$ due to the production and absorption processes of these neutrinos in the neutrino-cooling and heating regions; and (ii) a final dominance of the anisotropy of the radiated muon and tau neutrinos as a consequence of their asymmetric transport through the semi-transparent accretion layer, in which the total heavy-lepton neutrino luminosity hardly changes but the equator-to-pole asymmetry grows.

Our analysis therefore reveals that the gravitational-wave results for neutrinos depend on physical effects that are either not included or treated in a much simplified manner in previous works. It further reveals that a reliable and quantitatively meaningful computation of the tiny neutrino emission asymmetry is extremely difficult and does not only require a full transport treatment and good numerical resolution in all relevant regions, but ultimately must be done on the basis of 3D models.

\section{Summary and conclusions}

We have presented results of two stellar core-collapse simulations performed with the PROMETHEUS-VERTEX code for a nonrotating $15 M_{\odot}$ progenitor star in axial symmetry. One of these models was computed with a soft version of the Lattimer \& Swesty (1991) nuclear equation of state, and the other one with the stiffer Hillebrandt \& Wolff (1985) EoS, which produces a significantly less compact proto-neutron star. For these simulations we have compared the neutrino and gravitational-wave signals between core bounce and $400 \mathrm{~ms}$ later. In particular we were interested in signal features that may yield information about the action of the standing accretion shock instability (SASI) in the supernova core during the long phase of post-bounce accretion that precedes the onset of the supernova explosion in all recent multi-dimensional calculations of stellar core collapse.

Our main results can be summarized as follows:

1) In both simulations we found that the weakened bounce shock produces a negative entropy gradient that partly overlaps with the negative lepton number gradient left behind by the escaping shock breakout burst of electron neutrinos. This region is Ledoux unstable and develops prompt post-shock convection, which leads to an early burst of gravitationalwave emission lasting several ten milliseconds after core bounce. Its frequency spectrum shows a prominent peak around $70-100 \mathrm{~Hz}$.

2) A later, long-lasting phase of strong gravitational-wave emission with a long-time trend in growing wave amplitudes sets in at $t \gtrsim 100 \mathrm{~ms}$ after bounce. It is produced by nonradial mass motions and anisotropic neutrino emission that are caused by convective overturn inside the proto-neutron star and in the neutrino-heating layer behind the stalled shock, and in particular by vigorous sloshing of the accretion shock due to the development of low- $\ell$ mode SASI activity. The wave train for the matter signal consists of a superposition of quasi-periodic variations on timescales from a few milliseconds to several ten milliseconds. The spectra of the dominant $\ell=1,2$ SASI modes peak between about 20 and roughly $100 \mathrm{~Hz}$, and the gravitational-wave emission also shows significant power at frequencies up to $\sim 200 \mathrm{~Hz}$. The most prominent maxima of the gravitational-wave spectra, however, are located at higher frequencies of $300-800 \mathrm{~Hz}$ and originate mainly from the outer layers of the neutron star where rapid gas flows are triggered by the supersonic impact and deceleration of accretion funnels.

3) The high-frequency peak of the gravitational-wave spectrum and less clearly also the secondary maximum near the SASI frequency exhibit a dependence on the compactness of the nascent neutron star and thus on the properties of the high-density EoS: the more compact neutron star leads to more powerful shock oscillations and significantly larger gravitational-wave amplitudes earlier after core bounce. Also the characteristic frequencies of the low- $\ell$ SASI modes and of the gravitational-wave signal are higher. While the main peak of the wave spectrum is located at 
$300-600 \mathrm{~Hz}$ for the stiffer EoS, it can be found at 600$800 \mathrm{~Hz}$ for the softer EoS with the more compact remnant.

4) The more compact neutron star also radiates neutrinos with higher luminosities and greater mean energies. Anisotropic neutrino emission produces a low-frequency component of the gravitational-wave signal at $f \lessgtr 100-200 \mathrm{~Hz}$ with an amplitude that dominates the matter signal by up to a factor of two. The emission anisotropy is mostly established in the cooling and heating regions of the accretion layer outside of the neutrinospheres and is determined by the anisotropic transport of muon and tau neutrinos through this layer, which leads to a slightly higher flux of muon and tau neutrinos near the equatorial plane of the polar grid, i.e., perpendicular to the direction of the SASI oscillations of the supernova shock. Despite the stronger emission of $v_{\mathrm{e}}$ and $\bar{v}_{\mathrm{e}}$ from the accretion layer in the polar regions (i.e. in the direction of the SASI shock expansion and contraction), the gravitationalwave amplitude associated with the anisotropic energy loss in neutrinos exhibits a nearly monotonic trend to negative values.

5) The neutrino luminosities and mean energies, in particular the ones that can be received by an observer in the polar (and thus SASI) direction, show a quasi-periodic time variability with an amplitude of several ten percent (up to about 50\%) of the minimum values for the luminosities and of roughly $1 \mathrm{MeV}$ (up to $10 \%$ ) for the mean energies. The luminosity fluctuations are correlated and in phase with the energy variations and are somewhat bigger for $v_{\mathrm{e}}$ and $\bar{v}_{\mathrm{e}}$ than for heavylepton neutrinos. They originate from alternating phases of gas accumulation and compression in the accretion layer in particular around the poles, caused by the expansion and contraction of the shock in the course of the SASI oscillations. The frequency spectra of the neutrino luminosities show most power between about 20 and $200 \mathrm{~Hz}$ and significant power in a decaying tail up to about $400 \mathrm{~Hz}$ as a consequence of emission variability associated with faster, convective modulations of the accretion flow between supernova shock and nascent neutron star. Since SASI and convective activity occur inseparably in the gain layer and stir the cooling layer below, power is found to be distributed over a wide range of frequencies in the Fourier spectra of the luminosities.

The SASI induced temporal modulations of the luminosities and mean energies are features by which neutrinos can provide direct evidence of the dynamical processes that occur in the supernova core. Only few other cases are known where neutrinos carry such information, for example the prompt flash of electron neutrinos as a signature of the shock breakout from the neutrinosphere, or a possible second neutrino burst or abrupt termination of the neutrino emission, which are expected if the neutron star collapses to a more compact object or a black hole. A detection of the predicted characteristic, quasi-periodic variation in the neutrino event rate from a Galactic supernova would confirm the action of the SASI in the supernova core and would thus provide extremely valuable support of our present understanding of the core-collapse physics: the SASI is discussed as possible origin of global supernova asymmetries and pulsar kicks and as a potentially crucial agent on the route to the explosion.

Moreover, nonradial mass motions such as convection and the SASI in the supernova core after bounce produce gravitational-wave emission with significant intensity. We found that the wave spectrum exhibits a broad hump between about 20 and $200 \mathrm{~Hz}$ and a main maximum with clearly more power at considerably higher frequencies. The latter is associated with SASI and accretion induced gas flows in the neutron star surface layers, where much more mass is involved than in the fairly dilute post-shock region. Both the primary and the secondary maximum of the spectrum are sensitive to the structure of the neutron star: larger gravitational-wave amplitudes and correspondingly higher spectral maxima as well as higher frequencies are obtained with a softer equation of state and more compact remnant. The relative SASI variations of the neutrino luminosities do not appear to be very sensitive to the nuclear equation of state, and a clear EoS-dependent difference in the modulation frequencies is also not visible, but the absolute values of the luminosities and mean energies of the neutrinos radiated by the more compact and hotter, accreting proto-neutron star in our simulations are $10-20 \%$ higher.

Anisotropic neutrino emission is also an important source of gravitational waves with amplitudes that can dominate the matter signal. The corresponding spectrum possesses most power at frequencies below $100-200 \mathrm{~Hz}$. The emission anisotropy is a function with rapid short-time variability (with periods of milliseconds to $\gtrsim 10 \mathrm{~ms}$ ) and with an overlying, nearly monotonic long-time trend toward slightly stronger emission from equatorial regions, i.e., perpendicular to the direction of the SASI shock oscillations. This trend decides about the sign of the neutrino gravitational-wave amplitude. A detailed analysis of our models reveals that it is the result of complex transport physics in which the neutrinospheric flux, which has a low pole-to-equator anisotropy, is modified strongly in the inhomogeneous environment of the accretion layer around the forming neutron star. The pole-to-equator asymmetry changes with radius several times in both directions and the contributions from $v_{\mathrm{e}}, \bar{v}_{\mathrm{e}}$, and heavylepton neutrinos partly cancel each other. A reasonable determination of these transport asymmetries, which is not only important for gravitational-wave calculations but also for pulsar kick estimates based on neutrino recoil, requires detailed transport calculations. Even our sophisticated ray-by-ray approach may not be sufficient for accurate predictions of measurable effects, but a full multi-angle treatment of the transport may be necessary.

From this discussion it is clear that any reliable assessment of the detectability of SASI effects in the neutrino and gravitational-wave emission will ultimately require results from three-dimensional models. The growth of SASI modes with nonvanishing azimuthal order $m$ was shown to be of potential importance, and the presence of such nonaxisymmetric asymmetries can have a big influence on the structure and the dynamics of the accretion layer (Blondin \& Mezzacappa 2007; Yamasaki \& Foglizzo 2008; Iwakami et al. 2008). It is probable that also the emission asymmetries and time modulations of the emitted signals exhibit significant differences compared to 2D models, in particular because in 3D the SASI axis is not forced to coincide with an axis of symmetry and with the axis of the numerical grid.

The SASI and convective activity, its duration as well as strength, must be expected to vary with the progenitor star and to depend on the length of the postbounce accretion phase until the onset of the explosion (for hints to that, see the results for $11.2 M_{\odot}$ and $15 M_{\odot}$ explosions in Marek \& Janka 2007). In the nonlinear stage, the SASI and convective motions are of chaotic nature and therefore stochastic differences also in the large-scale pattern and global asymmetry are likely to develop from small initial differences even in progenitor stars (models) of the same kind (see, e.g., the different explosion asymmetries obtained in a large set of 2D simulations performed by Scheck et al. 2006). Nevertheless, basic features of the instabilites, in 
particular their mere presence and long-time growth behavior and the sizable modulation they impose on the emitted signals over a period of possibly hundreds of milliseconds, should be common to different realizations. Judged from our present understanding, however, a template-like uniformity of the signal structure with a well defined frequency (or frequency range) and amplitude that exhibit a regular dependence on the explosion and progenitor properties, appears not to be very likely. More model calculations are therefore needed to find out which signal properties such as the modulation frequency, are sufficiently generic to yield information about the neutron star equation of state.

\subsection{Detectability of SASI modulations}

Should the SASI signatures and their magnitude discussed in this work be confirmed by $3 \mathrm{D}$ results, the question arises whether they will be measurable with existing or emerging neutrino and gravitational-wave experiments in the case of the next Galactic supernova. A detailed prediction of supernova neutrino signals in terrestrial detectors is a highly complex problem, because the neutrinos radiated by the nascent neutron star undergo flavor conversions so that the flavor arriving at the Earth is not necessarily the same as the one leaving the source. On the one hand, the flavor evolution is affected by neutrino-matter oscillations during the neutrino propagation through the density gradients in the supernova mantle and envelope (where the $\mathrm{H}$ and $\mathrm{L}$ resonances are encountered at densities around $10^{3} \mathrm{~g} \mathrm{~cm}^{-3}$ and $10 \mathrm{~g} \mathrm{~cm}^{-3}$, respectively) and through the Earth. On the other hand, neutrino conversion is also induced by the nonlinear selfinteraction associated with neutrino-neutrino forward scattering in the dense neutrino background close to the neutron star (for an application of these phenomena in the context of the neutrino signals from oxygen-neon-magnesium core supernovae, see Lunardini et al. 2008, and from black hole formation in stellar collapse events, see Nakazato et al. 2008, for a discussion of the flavor conversion physics, see the references to original publications in the latter two papers). The flavor composition of the neutrino burst in the detector and thus the detector response therefore do not only depend on the type and structure of the progenitor star but also on a number of degrees of freedom associated with unknown neutrino properties and the location of the experiment, e.g. the neutrino-mass hierarchy (normal or inverted), the still undetermined mixing angle between the mass eigenstates 1 and 3, and the nadir angle of the neutrino path through the Earth.

Because of the involved complexity we defer an investigation of the flavor conversion effects and their consequences for the neutrino measurements to a future paper. However, since the SASI modulations affect the luminosities and mean energies of neutrinos and antineutrinos of all flavors with similar strength, we can ignore the flavor oscillation effects for a simple estimate to convince us that the SASI modulations of the radiated signal should be detectable in principle. Super-Kamiokande, for example, is expected to capture roughly 8000 electron antineutrinos through their absorption by protons, if a Galactic supernova occurs at a distance of $10 \mathrm{kpc}$ and releases at total energy of $5 \times 10^{52} \mathrm{erg}$ in $\bar{v}_{\mathrm{e}}$ with an average energy of $15 \mathrm{MeV}$. A luminosity of $4 \times 10^{52} \mathrm{erg} \mathrm{s}^{-1}$, which is a typical value for the emission maxima during the SASI phase, should account for an event rate of approximately $7000 \bar{v}_{\mathrm{e}}$ captures per second, corresponding to about 70 events in the $\sim 10 \mathrm{~ms}$ intervals of peak emission. If $L_{v}\left\langle\epsilon_{v}^{2}\right\rangle\left\langle\epsilon_{v}\right\rangle^{-1}$ is about $50 \%$ lower in luminosity minima, about one half of this event rate and event number can be expected during the low-emission periods. Therefore the SASI modulations will lead to a variability of the measured signal that can be larger, though not much, than the statistical $\sqrt{N_{v, \text { det }}}$ fluctuations, $N_{v \text {,det }}$ being the number of detected neutrinos in a time interval of $10 \mathrm{~ms}$. For Hyper-Kamiokande, whose fiducial volume would be roughly 30 times bigger than that of Super-Kamiokande, the Poisson noise would be reduced to a few percent of the event number within $10 \mathrm{~ms}$, so that the SASI modulations should create a clear signature. Even more powerful for this purpose is the IceCube experiment. It will be able to identify the supernova neutrino burst by the Cherenkov glow caused by the neutrino energy deposition in the ice, which will show up as time-correlated noise in all phototubes. For a supernova at a distance of $10 \mathrm{kpc}$, the $\bar{v}_{\mathrm{e}}$ luminosity maxima during the SASI phase are expected to cause a photon count rate of more than $10^{6}$ per second in the 4800 optical modules (see Dighe et al. 2003). The $\sim 1.2 \times 10^{4}$ events within $10 \mathrm{~ms}$ intervals and the variations of several 1000 events between periods of luminosity peaks and minima (in the case of IceCube these variations scale with $L_{v}\left\langle\epsilon_{v}^{3}\right\rangle\left\langle\epsilon_{v}\right\rangle^{-1}$, because the crucial quantity is the energy deposition in the detector) dominate by far the Poisson fluctuations associated with the background counting rate of $300 \mathrm{~Hz}$ per optical module; the Poisson variability of the background corresponds to $\sqrt{N_{\gamma}} \sim 120$ photon events in all phototubes, which is $\sim 1 \%$ of the supernova signal in a $10 \mathrm{~ms}$ interval (an impression of the detection capability even for a supernova model with a lower fluctuation amplitude of the neutrino emission than in the models discussed here, can be obtained from the upper lefthand panel of Fig. 2 in Dighe et al. 2003).

The detectability of gravitational-wave signals very similar to the ones presented in this work was investigated in much detail by Müller et al. (2004). Due to the restriction to a 90-degree grid (one hemisphere) in the case of the $15 M_{\odot}$ core-collapse model s15r considered in the latter paper, the contributions of dipolar SASI modes were not included in there. Moreover, since the collapse evolution was followed to later times in our most recent simulations (about $400 \mathrm{~ms}$ instead of $\sim 270 \mathrm{~ms}$ after bounce) and the quadrupole amplitudes exhibit a trend in rise during the later post-bounce phases, the signal-to-noise $(\mathrm{S} / \mathrm{N})$ ratios and maximum detection distances given for LIGO I and Advanced LIGO by Müller et al. (2004) are likely to be only lower limits. Since the S/N ratio scales roughly linearly with the Fourier transform of the wave amplitude, the detection prospects grow with longer integration periods of the signal and thus higher Fourier power. The measurement of dominant gravitational-wave power at frequencies of several hundred $\mathrm{Hz}$ with growing strength over a period of hundreds of milliseconds would therefore be a strong indication of SASI and convective activity around the nascent neutron star. Müller et al. (2004) estimated a S/N ratio of roughly 70 in Advanced LIGO for a $15 M_{\odot}$ supernova at $10 \mathrm{kpc}$, corresponding to the possibility of capturing gravitational waves from such sources up to a distance of roughly $100 \mathrm{kpc}$. A third-generation underground interferometer facility (Einstein Telescope) would lead to a gain in sensitivity of approximately a factor of 20 in the most relevant frequency range between $100 \mathrm{~Hz}$ and $1000 \mathrm{~Hz}$ (Spiering 2007). This would increase the expected detection rate of supernovae by up to a factor of 3 (Ando et al. 2005). A detailed analysis of the gravitationalwave signals and their observational implications from a greater sample of core-collapse models will be presented in a forthcoming paper.

Acknowledgements. We thank C.D. Ott and G.G. Raffelt for useful discussions. The project was supported by the Deutsche Forschungsgemeinschaft through the Transregional Collaborative Research Centers SFB/TR 27 "Neutrinos and Beyond" and SFB/TR 7 "Gravitational Wave Astronomy", and the Cluster of 
Excellence EXC 153 "Origin and Structure of the Universe" (http://www. universe-cluster.de). Computer time grants at the John von Neumann Institute for Computing (NIC) in Jülich, the Höchstleistungsrechenzentrum of the Stuttgart University (HLRS) under grant number SuperN/12758, the Leibniz-Rechenzentrum München, and the RZG in Garching are acknowledged.

\section{References}

Alexeyev, E. N., Alexeyeva, L. N., Krivosheina, I. V., \& Volchenko, V. I. 1988, Phys. Lett., B205, 209

Ando, S., Beacom, J. F., \& Yüksel, H. 2005, Phys. Rev. Lett., 95, 171101

Bionta, R. M., Blewitt, G., Bratton, C. B., et al. 1987, Phys. Rev. Lett., 58, 1494 (IMB collaboration)

Blanchet, L., Damour, T., \& Schäfer, G. 1990, MNRAS, 242, 289

Blondin, J. M., \& Mezzacappa, A. 2006, ApJ, 642, 401

Blondin, J. M., \& Mezzacappa, A. 2007, Nature, 445, 58

Blondin, J. M., Mezzacappa, A., \& DeMarino, C. 2003, ApJ, 584, 971

Bruenn, S. W., Dirk, C. J., Mezzacappa, A., et al. 2006, in SciDAC, Scientific Discovery through Advanced Computing, Denver, Colorado, USA, 25-29 June 2006, ed. W. M. Tang, et al., Journ. Phys. Conf. Ser., 46, 393 [arXiv:0709.0537]

Buras, R., Rampp, M., Janka, H.-Th., \& Kifonidis, K. 2006a, A\&A, 447, 1049

Buras, R., Janka, H.-Th., Rampp, M., \& Kifonidis, K. 2006b, A\&A, 457, 281

Burrows, A. 1987, ApJ, 318, L57

Burrows, A. 1988, ApJ, 334, 891

Burrows, A., \& Fryxell, B. A. 1993, ApJ, 418, L33

Burrows, A., Hayes, J., \& Fryxell, B. A. 1995, ApJ, 450, 830

Burrows, A., Livne, E., Dessart, L., Ott, C. D., \& Murphy, J. 2006, ApJ, 640, 878

Burrows, A., Livne, E., Dessart, L., Ott, C. D., \& Murphy, J. 2007a, ApJ, 655, 416

Burrows, A., Dessart, L., Livne, E., Ott, C. D., \& Murphy, J. 2007b, ApJ, 664, 416

Dessart, L., Burrows, A., Livne, E., \& Ott, C. D. 2006, ApJ, 645, 534

Dighe, A. S., Keil, M. Th., \& Raffelt, G. G. 2003, JCAP, 6, 005

Dimmelmeier, H., Ott, C. D., Marek, A., \& Janka, H.-Th. 2008, Phys. Rev. D, 78, 064056

Epstein, R. 1978, ApJ, 223, 1037

Epstein, R. 1979, MNRAS, 188, 305

Fischer, T., Gögelein, P., Liebendörfer, M., Mezzacappa, A., \& Thielemann, F.-K. 2008, in Origin of Matter and Evolution of Galaxies, ed. T. Suda T. Nozawa, A. Ohnishi, K. Kato, M. Y. Fujimoto, T. Kajino, \& S. Kubono, Amer. Inst. Phys. Conf. Proc., 1016, 277

Foglizzo, T. 2001, A\&A, 368, 311

Foglizzo, T. 2002, A\&A, 392, 353

Foglizzo, T., Scheck, L., \& Janka, H.-Th. 2006, ApJ, 652, 1436

Foglizzo, T., Galletti, P., Scheck, L., \& Janka, H.-Th. 2007, ApJ, 654, 1006

Fryer, C. L., \& Warren, M. S. 2002, ApJ, 574, L65

Fryer, C. L., \& Warren, M. S. 2004, ApJ, 601, 391

Fryer, C. L., Holz, D. E., \& Hughes, S. A. 2002, ApJ, 565, 430

Fryer, C. L., Holz, D. E., \& Hughes, S. A. 2004, ApJ, 609, 288

Heger, A., Woosley, S. E., \& Spruit, H. C. 2005, ApJ, 626, 350

Herant, M., Benz, W., Hix, W. R., Fryer, C. L., \& Colgate, S. A. 1994, ApJ, 435, 339
Hillebrandt, W., \& Wolff, R. G. 1985, in Nucleosynthesis: Challenges and New Developments, ed. W. D. Arnett, \& J. W. Truran (Chicago: Univ. Chicago Press), 131

Hillebrandt, W., Nomoto, K., \& Wolff, R. G. 1984, A\&A, 133, 175

Hirata, K., Kajita, T., Koshiba, M., Nakahata, M., Oyama, Y., et al. 1987, Phys. Rev. Lett., 58, 1490 (Kamiokande II collaboration)

Iwakami, W., Kotake, K., Ohnishi, N., Yamada, S., \& Sawada, K. 2008, ApJ, 678, 1207

Janka, H.-Th., \& Müller, E. 1996, A\&A, 306, 167

Janka, H.-Th., Buras, R., Kitaura, F. S., et al. 2005, Nucl. Phys., A758, 19c

Janka, H.-Th., Langanke, K., Marek, A., Martínez-Pinedo, G., \& Müller, B. 2007, Phys. Rep., 442, 38

Keil, W., \& Janka, H.-Th. 1995, A\&A, 296, 145

Keil, W., Janka, H.-Th., \& Müller, E. 1996, ApJ, 473, L111

Keil, W., Raffelt, G., \& Janka, H.-Th. 2003, ApJ, 590, 971

Kotake, K., Sato, K., \& Takahashi, K. 2006, Rep. Prog. Phys., 69, 971

Kotake, K., Ohnishi, N., \& Yamada, S. 2007, ApJ, 655, 406

Lattimer, J. M., \& Swesty, F. D. 1991, Nucl. Phys., A535, 331

Liebendörfer, M., Mezzacappa, A., Messer, O. E. B., et al. 2003, Nucl. Phys., A719, 144c

Lunardini, C., Müller, B., \& Janka, H.-Th. 2008, Phys. Rev. D, 78, 023016

Marek, A. 2007, Ph.D. Thesis, Technische Universität München

Marek, A., \& Janka, H.-Th. 2007, ApJ, in press [arXiv:0708.3372]

Marek, A., Dimmelmeier, H., Janka, H.-Th., Müller, E., \& Buras, R. 2006, A\&A, 445,273

Müller, E., \& Janka, H.-Th. 1997, A\&A, 317, 140

Müller, E., Rampp, M., Buras, R., Janka, H.-Th., \& Shoemaker, D. H. 2004, ApJ, 603, 221

Murphy, J. W., \& Burrows, A. 2008, ApJ, 688, 1159

Nakazato, K., Sumiyoshi, K., Suzuki, H., \& Yamada, S. 2008, Phys. Rev. D, 78, 083014

Ohnishi, N., Kotake, K., \& Yamada, S. 2006, ApJ, 641, 1018

Ott, C. D. 2008, Classical and Quantum Gravity, submitted [arXiv: 0809. 0695]

Ott, C. D., Burrows, A., Livne, E., \& Walder, R. 2004, ApJ, 600, 834

Ott, C. D., Burrows, A., Dessart, L., \& Livne, E. 2006, Phys. Rev. Lett., 96, 201102

Ott, C. D., Burrows, A., Dessart, L., \& Livne, E. 2008, ApJ, 685, 1069

Raffelt, G. G. 2001, ApJ, 561, 890

Rampp, M., \& Janka, H.-Th. 2002, A\&A, 396, 361

Sagert, I., Hempel, M., Pagliara, G., et al. 2008, Phys. Rev. Lett., submitted

Scheck, L., Plewa, T., Janka, H.-Th., Kifonidis, K., \& Müller, E. 2004, Phys. Rev. Lett., 92, 011103

Scheck, L., Kifonidis, K., Janka, H.-Th., \& Müller, E. 2006, A\&A, 457, 963

Scheck, L., Janka, H.-Th., Foglizzo, T., \& Kifonidis, K. 2008, A\&A, 477, 931

Shen, H., Toki, H., Oyamatsu, K., \& Sumiyoshi, K. 1998, Nucl. Phys., A637, 435

Spiering, C. 2007, The Messenger, 129, 33

Sumiyoshi, K., Yamada, S., Suzuki, H., \& Chiba, S. 2006, Phys. Rev. Lett., 97, 091101

Sumiyoshi, K., Yamada, S., \& Suzuki, H. 2007, ApJ, 667, 382

Swesty, F. D., \& Myra, E. S. 2005, J. Phys. Conf. Ser., 16, 380

Thompson, T. A., Quataert, E., \& Burrows, A. 2005, ApJ, 620, 861

Yamasaki, T., \& Foglizzo, T. 2008, ApJ, 679, 607

Woosley, S. E., \& Bloom, J. S. 2006, ARA\&A, 44, 507

Woosley, S. E., \& Weaver, T. A. 1995, ApJS, 101, 181 